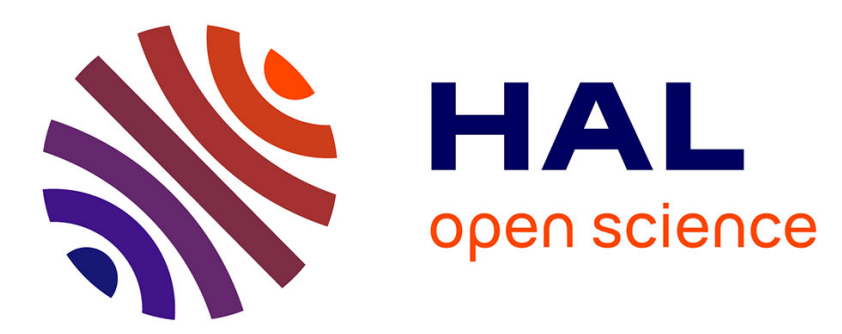

\title{
Stability estimate for an inverse problem for the electro-magnetic wave equation and spectral boundary value problem
}

Hajer Ben Joud

\section{- To cite this version: \\ Hajer Ben Joud. Stability estimate for an inverse problem for the electro-magnetic wave equation and spectral boundary value problem. 2009. hal-00426786}

\section{HAL Id: hal-00426786 \\ https://hal.science/hal-00426786}

Preprint submitted on 27 Oct 2009

HAL is a multi-disciplinary open access archive for the deposit and dissemination of scientific research documents, whether they are published or not. The documents may come from teaching and research institutions in France or abroad, or from public or private research centers.
L'archive ouverte pluridisciplinaire HAL, est destinée au dépôt et à la diffusion de documents scientifiques de niveau recherche, publiés ou non, émanant des établissements d'enseignement et de recherche français ou étrangers, des laboratoires publics ou privés. 


\title{
Stability estimate for an inverse problem for the electro-magnetic wave equation and spectral boundary value problem
}

\author{
Hajer Ben Joud ${ }^{1}$ \\ ${ }^{1}$ Department of Mathematics, \\ Faculty of Sciences of Bizerte, \\ 7021 Jarzouna Bizerte, Tunisia. \\ mourad.bellassoued@fsb.rnu.tn
}

\begin{abstract}
In this paper, we prove the stability estimate of the inverse problem for the determination the magnetic field and the electric potential using the Neumann spectral data. We show that the knowledge of the eigenvalues $\left\{\lambda_{k}, k \geq 1\right\}$ and the boundary value of the normal derivatives of the corresponding eigenfunctions $\left\{\partial_{\nu} \varphi_{k}, k \geq 1\right\}$, and of the electro-magnetic Schrödinger operator, are sufficient to uniquely determine the magnetic field and the electric potential.

To obtain this result, we establish the stability estimate of the inverse problem of determining the electric potential entering the electro-magnetic wave equation in a bounded smooth domain in $\mathbb{R}^{d}$ from boundary observations. This information is enclosed in the hyperbolic (dynamic) Dirichletto-Neumann map associated to the solutions to the electro-magnetic wave equation. We prove in dimension $d \geq 2$ that the knowledge of the Dirichlet to Neumann map for the electro-magnetic wave equation measured on the boundary determines uniquely the electric potential.
\end{abstract}

Keywords: Stability estimate, hyperbolic inverse problem, magnetic field, Dirichlet to Neumann map, spectral data.

\section{Introduction}

Throughout this paper, we assume that the dimension $d \geq 2$. Let $\Omega \subset \mathbb{R}^{d}$ be an open bounded set, with $\mathcal{C}^{\infty}$ boundary $\Gamma=\partial \Omega$. Given $T>0$, we consider the following initial boundary value problem for the wave equation with a magnetic and electric potential

$$
\begin{cases}\left(\partial_{t}^{2}-\Delta_{A}+q(x)\right) u(t, x)=0 & \text { in } Q=(0, T) \times \Omega, \\ u(0, x)=0, \quad \partial_{t} u(0, x)=0 & \text { in } \Omega, \\ u(t, x)=f(t, x) & \text { on } \Sigma=(0, T) \times \Gamma,\end{cases}
$$

where

$$
\Delta_{A}=\sum_{j=1}^{d}\left(\partial_{j}+i a_{j}\right)^{2}=\Delta+2 i A \cdot \nabla+i \operatorname{div}(A)-A \cdot A
$$


and $A=\left(a_{j}\right)_{1 \leq j \leq d} \in W^{3, \infty}\left(\Omega ; \mathbb{R}^{d}\right)$ is a magnetic potential and the bounded electric potential $q \in$ $L^{\infty}(\Omega)$. It is well known (see [24]) that if $f(0, x)=0$ then (1.1) is a well-posed initial-boundary value problem. Therefore, we may define the operator

$$
\begin{aligned}
\Lambda_{A, q}: H^{1}(\Sigma) & \longrightarrow L^{2}(\Sigma) \\
f & \longmapsto\left(\partial_{\nu}+i A \cdot \nu\right) u
\end{aligned}
$$

where $\nu(x)$ denotes the unit outward normal to $\Gamma$ at $x$. The operator $\Lambda_{A, q}$, which is the main subject of this paper, is called the Dirichlet to Neumann map of (1.1) on $\Sigma$.

Using energy estimate one can prove that $\Lambda_{A, q}$ is continuous from $H^{1}(\Sigma)$ to $L^{2}(\Sigma)$. The inverse problem is whether knowledge of the Dirichlet-to-Neumann map $\Lambda_{A, q}$ on the boundary determines uniquely the magnetic potential $A$ and the electric potential $q$.

As given in [3] and [12], it is clear that one can not hope to uniquely determine the vector field $A$ and that is due to the invariance of the Dirichlet-to-Neumann map by gauge transformation. For that, in geometric term, the vector field $A$ defines the connection given by the one form $\alpha_{A}=\sum_{j=1}^{d} a_{j} d x_{j}$, and the non-uniqueness says that the best we could hope to reconstruct from the Dirichlet-to-Neumann map $\Lambda_{A, q}$ is the connection $d \alpha_{A}$ given by

$$
d \alpha_{A}=\sum_{i, j=1}^{d}\left(\frac{\partial a_{i}}{\partial x_{j}}-\frac{\partial a_{j}}{\partial x_{i}}\right) d x_{j} \wedge d x_{i} .
$$

We denote by

$$
\mathscr{H}_{A, q}(x, D)=-\Delta_{A}+q(x)
$$

where $\Delta_{A}$ as given by (1.2), with domain $D\left(\mathscr{H}_{A, q}\right)=H_{0}^{1}(\Omega) \cap H^{2}(\Omega)$. It is well known that the spectrum of $\mathscr{H}_{A, q}$ consists of a sequence of the eigenvalues, counted according to their multiplicities

$$
\lambda_{1, A, q} \leq \lambda_{2, A, q} \leq \ldots \leq \lambda_{k, A, q} \rightarrow+\infty .
$$

The corresponding eigenfunctions is denoted by $\left(\varphi_{k, A, q}\right)$. We may assume that this sequence form an orthonormal basis of $L^{2}(\Omega)$.

We consider the eigenvalue problem

$$
\begin{cases}\mathscr{H}_{A_{l}, q_{l}}(x, D) \varphi(x)=\lambda \varphi(x) & \text { in } \Omega, \\ \varphi(x)=0 & \text { on } \Gamma .\end{cases}
$$

To simplify the notation we have

$$
\left(\lambda_{k, A_{l}, q_{l}}\right)_{k}=\left(\lambda_{k}^{l}\right)_{k} \text { and }\left(\varphi_{k, A_{l}, q_{l}}\right)_{k}=\left(\varphi_{k}^{l}\right)_{k} \quad \text { for } l=1,2 .
$$

We assume that 0 is not an eigenvalue of $\mathscr{H}_{A, q}$. Then using the elliptic regularity, we obtain

$$
\left\|\varphi_{k}\right\|_{H^{2}(\Omega)} \leq C \lambda_{k}\left\|\varphi_{k}\right\|_{L^{2}(\Omega)} .
$$

Where, $C$ is depending only on $\Omega, M$. Therefore

$$
\left\|\partial_{\nu} \varphi_{k}\right\|_{H^{1 / 2}(\Gamma)} \leq C \lambda_{k} .
$$


To obtain an asymptotic behavior of eigenvalues of $\mathscr{H}_{A, q}$, we cannot apply the Weyl's formula. Then this is owing to the difficulty to find an exact elementary solution of the parabolic equation corresponds to this operator. Since the counting function

$$
N(\tilde{\lambda})=\#\left\{k \geq 1, \lambda_{k} \leq \tilde{\lambda}\right\}
$$

depends only on principal part of the symbol of $\mathscr{H}_{A, q}(x, D)$ which equal to $|\xi|^{2}$ (See Reiko and Sigeru [30]), we have

$$
C^{-1} k^{2 / d} \leq \lambda_{k} \leq C k^{2 / d} .
$$

Then, we can conclude that

$$
\left\|\partial_{\nu} \varphi_{k}\right\|_{H^{1 / 2}(\Gamma)} \leq C k^{2 / d} .
$$

We recall that $\ell^{1}$ is the usual Banach space of real-valued sequences such that the corresponding series is absolutely convergent. This space is equipped with its natural norm.

We fix $d+2>m>\frac{d}{2}+1$ such that $\mathbf{w}=\left(\mathbf{w}_{k}\right)_{k}$ is the sequence given by $\mathbf{w}_{k}=k^{\frac{-2 m}{d}}$ for each $k \geq 1$. We introduce the following Banach spaces

$$
\ell_{\mathbf{w}}^{1}\left(H^{1 / 2}(\Gamma)\right)=\left\{g=\left(g_{k}\right)_{k} ; g_{k} \in H^{1 / 2}(\Gamma) ; k \geq 1 \text { and }\left(\mathbf{w}_{k}\left\|g_{k}\right\|_{H^{1 / 2}(\Gamma)}\right) \in \ell^{1}\right\},
$$

and

$$
\ell_{\mathbf{w}}^{1}(\mathbb{C})=\left\{y=\left(y_{k}\right)_{k} ; \quad y_{k} \in \mathbb{C} ; \quad k \geq 1 \text { and }\left(\mathbf{w}_{k}\left|y_{k}\right|\right) \in \ell^{1}\right\} .
$$

The natural norms on this spaces are respectively

$$
\|g\|_{\ell_{\mathbf{w}}^{1}\left(H^{1 / 2}(\Gamma)\right)}=\sum_{k \geq 1} \mathbf{w}_{k}\left\|g_{k}\right\|_{H^{1 / 2}(\Gamma)}
$$

and

$$
\|y\|_{\ell_{\mathbf{w}}^{1}(\mathbb{C})}=\sum_{k \geq 1} \mathbf{w}_{k}\left|y_{k}\right|
$$

In what follows, we shall use the following notations:

$$
D_{\Omega}=\inf \left\{R \in \mathbb{R}^{+}: \Omega \subset B\left(x_{0}, R\right) \text { for some } \quad x_{0} \in \mathbb{R}^{d}\right\} .
$$

Here $B\left(x_{0}, R\right)=\left\{x \in \mathbb{R}^{d}:\left|x-x_{0}\right|<R\right\}$.

The one-dimensional inverse problem of the reconstruction of a differential operator from its spectral data goes back to 40-50 th (Börg [10], Levinson [23], Gelfand-Levitan [14], Krein [20, 21]).

The issue of stability estimates for multidimensional inverse spectral problems for hyperbolic operator with electric potential was first addressed by Alessandrini and Sylvester [1] and recently Bellassoued, Choulli and Yamamoto [4] found a stability estimate related to the multidimensional Borg-Levinson theorem using a result of stability in determining $q$ from a partial Dirichlet to Neumann map provided that $q$ is a priori known in a neighborhood of the boundary of spatial domain and satisfies on additional condition. And this result is an extension of result in [11] which itself is a variant of a theorem in [1].

The inverse spectral problem for the Schrödinger operator with analytic potential was considered by Berezanskii [8, 9]. In 1987 Sylvester and Uhlmann [33] and Novikov and Henkin [15] proved the 
uniqueness in the nonanalytic case. A.Nachman, J.Sylvester, G.Uhlmann [25] solved the inverse boundary spectral problem for the Schrödinger operator by reducing it to the inverse boundary value problem in fixed frequency and then using the method of complex geometric optics [33].

For more general literature see Katchalov, Kurylev and Lassas [22] studies of the inverse boundary spectral problem. The main aim of this book is to develop a rigorous theory to solve several types of inverse problem exactly, rather than to discuss applied numerical aspects of these problems.

In this paper, we show how to obtain estimates for $d \alpha_{A}$ and $q$ by some spectral data. More precisely, we will show that the knowledge of the eigenvalues $\left\{\lambda_{k}, k \geq 1\right\}$ and the boundary value of the normal derivatives of the corresponding eigenfunctions $\left\{\partial_{\nu} \varphi_{k}, k \geq 1\right\}$ is sufficient to uniquely determine the magnetic field $d \alpha_{A}$ and the electric potential $q$. Our main result is given by the following theorem

Theorem 1 Let $A_{1}, A_{2}$ be two real $W^{3, \infty}\left(\Omega ; \mathbb{R}^{d}\right)$ vector fields on $\Omega$ and $q_{1}, q_{2} \in L^{\infty}(\Omega), \alpha>\frac{d}{2}+3$ such that $\left\|A_{l}\right\|_{H^{\alpha}(\Omega)} \leq M$ and $\left\|q_{l}\right\|_{L^{\infty}(\Omega)} \leq M$ for $l=1,2$. We assume that $A_{1}=A_{2}$ and $q_{1}=q_{2}$ on the boundary $\Gamma$. Then there exist a constant $C>0$ and $0<\theta<1$ such that

$$
\left\|q_{1}-q_{2}\right\|_{H^{-1}(\Omega)}+\left\|d \alpha_{A_{1}}-d \alpha_{A_{2}}\right\|_{L^{\infty}(\Omega)} \leq C\left(\left\|\lambda^{1}-\lambda^{2}\right\|_{\ell_{w}^{1}(\mathbb{C})}+\left\|\partial_{\nu} \varphi^{1}-\partial_{\nu} \varphi^{2}\right\|_{\ell_{w}^{1}\left(H^{1 / 2}(\Gamma)\right)}\right)^{\theta} .
$$

The left-hand side of the inequality is assumed to be small and $C$ depends on $\Omega, M$ and $d$.

To prove Theorem 1, we shall make use of the Dirichlet to Neumann given by (1.3). So we are going to establish a stability result for the inverse problem consisting in the determination of magnetic field $d \alpha_{A}$ and the potential $q$ from the norm of the D-to-N map $\Lambda_{A, q}$ in $\mathcal{L}\left(H^{1}(\Sigma), L^{2}(\Sigma)\right)$.

Theorem 2 Let $A_{1}, A_{2}$ be two real $W^{3, \infty}\left(\Omega ; \mathbb{R}^{d}\right)$ vector fields on $\Omega$ and $q_{1}, q_{2} \in L^{\infty}(\Omega), \alpha>\frac{d}{2}+3$ such that $\left\|A_{l}\right\|_{H^{\alpha}(\Omega)} \leq M$ and $\left\|q_{l}\right\|_{L^{\infty}(\Omega)} \leq M$ for $l=1,2$. We assume that $A_{1}=A_{2}$ and $q_{1}=q_{2}$ on the boundary $\Gamma$ and $T>D_{\Omega}$. Then there exist a constant $C>0$ and $\mu^{\prime} \in(0,1)$ such that

$$
\left\|q_{1}-q_{2}\right\|_{H^{-1}(\Omega)}+\left\|d \alpha_{A_{1}}-d \alpha_{A_{2}}\right\|_{L^{\infty}(\Omega)} \leq C\left\|\Lambda_{A_{1}, q_{1}}-\Lambda_{A_{2}, q_{2}}\right\|^{\mu^{\prime}} .
$$

Where $C$ depends on $\Omega, M, T$ and $d$.

In order to study the spectral stability, we denote by $\tilde{\Lambda}_{A, q}$ the restriction of $\Lambda_{A, q}$ in the space $H^{2 d+4}\left(0, T ; H^{3 / 2}(\Gamma)\right)$ which given by

$$
\Lambda_{A, q}^{\sharp}: H^{2 d+4}\left(0, T ; H^{3 / 2}(\Gamma)\right) \longrightarrow L^{2}\left((0, T) ; H^{s}(\Gamma)\right),
$$

for any $s \in[0,1 / 2]$. We denote $\|.\|_{s}$ the norm in $\mathcal{L}\left(H^{2 d+4}\left(0, T ; H^{3 / 2}(\Gamma)\right), L^{2}\left((0, T) ; H^{s}(\Gamma)\right)\right)$.

Theorem 3 Let $A_{1}, A_{2}$ be two real $W^{3, \infty}\left(\Omega ; \mathbb{R}^{d}\right)$ vector fields on $\Omega$ and $q_{1}, q_{2} \in L^{\infty}(\Omega), \alpha>\frac{d}{2}+3$ such that $\left\|A_{l}\right\|_{H^{\alpha}(\Omega)} \leq M$ and $\left\|q_{l}\right\|_{L^{\infty}(\Omega)} \leq M$ for $l=1,2$. We assume that $A_{1}=A_{2}$ and $q_{1}=q_{2}$ on the boundary $\Gamma$ and $T>D_{\Omega}$. Then there exists a constant $C>0$ and $\mu \in(0,1)$ such that

$$
\left\|q_{1}-q_{2}\right\|_{H^{-1}(\Omega)}+\left\|d \alpha_{A_{1}}-d \alpha_{A_{2}}\right\|_{L^{\infty}(\Omega)} \leq C\left\|\Lambda_{A_{1}, q_{1}}^{\sharp}-\Lambda_{A_{2}, q_{2}}^{\sharp}\right\|_{s}^{\mu} .
$$

Where $C$ depends on $\Omega, M, T$ and $d$. 
The inverse problem given by Theorem 2 and 3 is to recover information about the magnetic and electric potential from the D-to-N map measured on the whole boundary.

The hyperbolic inverse problem often occurs in applications, they have been extensively studied and there are several methods to solve them. Most methods are based on the geometrical ideas and finite speed of propagation e.g Isakov [18]. The results devoted to the uniqueness in these problems can be found in Eskin [13], Rakesh-Symes [28], Ramm-Sjostrand [29].

Although stability in the hyperbolic inverse problem is a less studied subject than uniqueness, there are already many interesting results in this direction. These results Bellassoued [2], Bellassoued-Ben Joud [3], Bellassoued-Jellali-Yamamoto [5, 6], Imanuvilov-Yamamoto [16], [19], [32].

The paper is organized as follows. Section 2 is devoted to prove the Theorem 1. Section 3 deals with the construction of geometrical optics solutions, Hodge decomposition and contains the proof of Theorem 2 and 3. Finally, we give a proof of some important Lemmas considered in the Appendix.

\section{Proof of Theorem 1}

Let $A \in W^{3, \infty}\left(\Omega, \mathbb{R}^{d}\right)$ and $q \in L^{\infty}(\Omega)$. We denote $\sigma\left(\mathscr{H}_{A, q}\right)=\left\{\lambda_{k}\right\}_{k \geq 1}$ be the spectrum of $\mathscr{H}_{A, q}$ and $\rho\left(\mathscr{H}_{A, q}\right)=\mathbb{C} \backslash \sigma\left(\mathscr{H}_{A, q}\right)$ be resolvent set of $\sigma\left(\mathscr{H}_{A, q}\right)$. For any $\lambda \in \rho\left(\mathscr{H}_{A, q}\right)$ and $f \in H^{3 / 2}(\Gamma)$, we introduce the elliptic problem

$$
\begin{cases}\left(-\Delta_{A}+q-\lambda\right) u=0 & \text { in } \Omega \\ u=f & \text { on } \Gamma .\end{cases}
$$

We define the D-to-N map associated to the above problem given by

$$
\Pi_{A, q}: H^{3 / 2}(\Gamma) \rightarrow H^{s}(\Gamma)
$$

for $0<s<\frac{1}{2}$, such that $\Pi_{A, q}(f)=\frac{\partial u}{\partial \nu}+i A . \nu u$. We denote by $\|\cdot\|_{\frac{3}{2}, s}$ the norm in $\mathcal{L}\left(H^{3 / 2}(\Gamma), H^{s}(\Gamma)\right)$.

We are going to introduce the following lemmas, which are given in [1], [4] and [11], in case we have a laplace instead of the magnetic laplace. For more detail, we shall sketch the proofs to Lemma 2.2 and 2.3 in an appendix.

Lemma 2.1 Let $A \in W^{3, \infty}\left(\Omega ; \mathbb{R}^{d}\right)$ and $q \in L^{\infty}(\Omega)$. Then for any $m>\frac{d}{2}, f \in H^{3 / 2}(\Gamma)$ and $\lambda \in$ $\rho\left(\mathscr{H}_{A, q}\right)$, we have

$$
\frac{d^{m}}{d \lambda^{m}}\left(\Pi_{A, q}(\lambda)\right) f=-\left.m ! \sum_{k \geq 1} \frac{1}{\left(\lambda_{k}-\lambda\right)^{m+1}}\left\langle f, \partial_{\nu} \varphi_{k}\right\rangle \partial_{\nu} \varphi_{k}\right|_{\Gamma}
$$

where $\langle$,$\rangle denote the inner product in L^{2}(\Gamma)$.

Lemma 2.2 Let $0<s<\frac{1}{2}$. We fix $j \geq 0$ then we have

$$
\left\|\left(\frac{d}{d \lambda}\right)^{j}\left(\Pi_{A_{1}, q_{1}}(\lambda)-\Pi_{A_{2}, q_{2}}(\lambda)\right)\right\|_{\frac{3}{2}, s} \leq C|\lambda|^{-j+\frac{1}{4}(1+2 s)} .
$$


Remark 1 The difference between the result of the previous lemma and what was expressed in [1] is the power of the $\lambda$ and this is due to the presence of the magnetic potential.

Lemma 2.3 For any $f \in H^{2(d+2)}\left(0, T, H^{1 / 2}(\Gamma)\right)$ satisfies

$$
\left(\frac{\partial}{\partial t}\right)^{j} f(0, x)=0 \text { for } x \in \Gamma \text { and } j=0,1, \ldots, 2 d+3,
$$

we have

$$
\Lambda_{A, q}^{\sharp}(f)=\sum_{j=0}^{d+1}\left[\left(\frac{d}{d \lambda}\right)^{j} \Pi_{A, q}(\lambda)\right]_{\lambda=0}\left(-\frac{\partial^{2}}{\partial t^{2}}\right)^{j} f+\mathscr{R}_{A, q} f,
$$

where,

$$
\mathscr{R}_{A, q}(f)=\sum_{k=1}^{\infty}\left(\lambda_{k}\right)^{-d-5 / 2} \frac{\partial \varphi_{k}}{\partial \nu} \int_{0}^{t} \sin \sqrt{\lambda_{k}}(t-s)\left\langle\left(-\frac{\partial^{2}}{\partial s^{2}}\right)^{d+2} f(s, .), \partial_{\nu} \varphi_{k}(.)\right\rangle d s+i A . \nu f,
$$

where $\langle$,$\rangle denote the inner product in L^{2}(\Gamma)$.

\subsection{Preliminaries estimations}

In this subsection, We will introduce the estimates, which are the keys of our result. For that we note

$$
\begin{gathered}
\mathscr{P}^{(j)}(\lambda)=\left(\frac{d}{d \lambda}\right)^{j}\left(\Pi_{A_{1}, q_{1}}(\lambda)-\Pi_{A_{2}, q_{2}}(\lambda)\right), \\
\mathscr{R}^{\sharp}(f)=\mathscr{R}_{A_{1}, q_{1}}(f)-\mathscr{R}_{A_{2}, q_{2}}(f)
\end{gathered}
$$

and

$$
\delta=\left\|\lambda^{1}-\lambda^{2}\right\|_{\ell_{\mathbf{w}}^{1}(\mathbb{C})}+\left\|\partial_{\nu} \varphi^{1}-\partial_{\nu} \varphi^{2}\right\|_{\ell_{\mathbf{w}}^{1}\left(H^{1 / 2}(\Gamma)\right)} .
$$

Lemma 2.4 There exists a constant $C>0$ such that the following estimates holds true

$$
\left\|\mathscr{P}^{(d+1)}(\lambda)\right\|_{\frac{3}{2}, s} \leq C \delta .
$$

and

$$
\left\|\mathscr{P}^{(j)}(0)\right\|_{\frac{3}{2}, s} \leq C \delta^{\theta}
$$

for any $\lambda \leq 0$. Where $\theta$ is equal to $1-\frac{4 d-4}{4 d+5-2 s}$. 
Proof . We first address the case where $j=d+1$. We suppose that $A_{1}=A_{2}$ on $\Gamma$. For $f \in H^{3 / 2}(\Omega)$, we have the following equation

$$
\begin{aligned}
\mathscr{P}^{(d+1)}(\lambda)(f)= & (d+1) !\left(-\sum_{k \geq 1} \frac{1}{\left(\lambda_{k}^{1}-\lambda\right)^{d+2}}\left\langle f, \partial_{\nu} \varphi_{k}^{1}\right\rangle \partial_{\nu} \varphi_{k}^{1}+\sum_{k \geq 1} \frac{1}{\left(\lambda_{k}^{2}-\lambda\right)^{d+2}}\left\langle f, \partial_{\nu} \varphi_{k}^{2}\right\rangle \partial_{\nu} \varphi_{k}^{2}\right) \\
= & -(d+1) ! \sum_{k \geq 1}\left(\frac{1}{\left(\lambda_{k}^{1}-\lambda\right)^{d+2}}-\frac{1}{\left(\lambda_{k}^{2}-\lambda\right)^{d+2}}\right)\left\langle f, \partial_{\nu} \varphi_{k}^{1}\right\rangle \partial_{\nu} \varphi_{k}^{1} \\
& -(d+1) ! \sum_{k \geq 1} \frac{1}{\left(\lambda_{k}^{2}-\lambda\right)^{d+2}}<f, \partial_{\nu} \varphi_{k}^{1}-\partial_{\nu} \varphi_{k}^{2}>\partial_{\nu} \varphi_{k}^{1} \\
& -(d+1) ! \sum_{k \geq 1} \frac{1}{\left(\lambda_{k}^{2}-\lambda\right)^{d+2}}\left(\left\langle f, \partial_{\nu} \varphi_{k}^{2}\right\rangle\left(\partial_{\nu} \varphi_{k}^{1}-\partial_{\nu} \varphi_{k}^{2}\right)\right) \\
= & \mathscr{I}_{1}+\mathscr{I}_{2}+\mathscr{I}_{3} .
\end{aligned}
$$

Where

$$
\begin{gathered}
\mathscr{I}_{1}=-(d+1) ! \sum_{k \geq 1}\left(\frac{1}{\left(\lambda_{k}^{1}-\lambda\right)^{d+2}}-\frac{1}{\left(\lambda_{k}^{2}-\lambda\right)^{d+2}}\right)\left\langle f, \partial_{\nu} \varphi_{k}^{1}\right\rangle \partial_{\nu} \varphi_{k}^{1} \\
\mathscr{I}_{2}=-(d+1) ! \sum_{k \geq 1} \frac{1}{\left(\lambda_{k}^{2}-\lambda\right)^{d+2}}\left\langle f, \partial_{\nu} \varphi_{k}^{1}-\partial_{\nu} \varphi_{k}^{2}\right\rangle \partial_{\nu} \varphi_{k}^{1} \\
\mathscr{I}_{3}=-(d+1) ! \sum_{k \geq 1} \frac{1}{\left(\lambda_{k}^{2}-\lambda\right)^{d+2}}\left(\left\langle f, \partial_{\nu} \varphi_{k}^{2}\right\rangle\left(\partial_{\nu} \varphi_{k}^{1}-\partial_{\nu} \varphi_{k}^{2}\right)\right) .
\end{gathered}
$$

We have the following estimates

$$
\left\|\mathscr{I}_{1}\right\|_{H^{1 / 2}(\Gamma)} \leq(d+1) ! \sum_{k \geq 1}\left|\frac{1}{\left(\lambda_{k}^{1}-\lambda\right)^{d+2}}-\frac{1}{\left(\lambda_{k}^{2}-\lambda\right)^{d+2}}\right|\left\|\partial \varphi_{k}^{1}\right\|_{H^{1 / 2}(\Gamma)}^{2}\|f\|_{L^{2}(\Gamma)} .
$$

Using the asymptotic behavior of the eigenvalue and elliptic regularity we have

$$
\begin{aligned}
\left|\frac{1}{\left(\lambda_{k}^{1}-\lambda\right)^{d+2}}-\frac{1}{\left(\lambda_{k}^{2}-\lambda\right)^{d+2}}\right| & \leq C \max _{k \geq 1}\left(\frac{1}{\left(\lambda_{k}^{1}\right)^{d+3}}, \frac{1}{\left(\lambda_{k}^{2}\right)^{d+3}}\right)\left|\lambda_{k}^{1}-\lambda_{k}^{2}\right| \\
& \leq \frac{C}{k^{\frac{2}{d}(d+3)}}\left|\lambda_{k}^{1}-\lambda_{k}^{2}\right|
\end{aligned}
$$

and

$$
\left\|\partial \varphi_{k}^{1}\right\|_{H^{1 / 2}(\Gamma)} \leq k^{\frac{2}{d}}
$$

Combining (2.6), (2.7) and (2.8) we obtain

$$
\left\|\mathscr{I}_{1}\right\|_{H^{1 / 2}(\Gamma)} \leq(d+1) ! \sum_{k \geq 1} \frac{1}{k^{\frac{2}{d}(d+2)}}\left|\lambda_{k}^{1}-\lambda_{k}^{2}\right|\|f\|_{L^{2}(\Gamma)} .
$$

Using the fact that $\frac{2}{d}(d+2)>\frac{2}{d} m$, then we have

$$
\left\|\mathscr{I}_{1}\right\|_{H^{1 / 2}(\Gamma)} \leq C\left\|\lambda^{1}-\lambda^{2}\right\|_{\ell_{\mathbf{w}}^{1}(\mathbb{C})}\|f\|_{L^{2}(\Gamma)} .
$$


Using expression of $\mathscr{I}_{2}$, we have

$$
\left\|\mathscr{I}_{2}\right\|_{H^{1 / 2}(\Gamma)} \leq(d+1) ! \sum_{k \geq 1} \frac{1}{\left|\lambda_{k}^{2}-\lambda\right|^{d+2}}\left\|\partial_{\nu} \varphi_{k}^{1}\right\|_{H^{1 / 2}(\Gamma)}\left\|\partial_{\nu} \varphi_{k}^{1}-\partial_{\nu} \varphi_{k}^{2}\right\|_{H^{1 / 2}(\Gamma)}\|f\|_{L^{2}(\Gamma)} .
$$

By (2.8) we have

$$
\begin{aligned}
\left\|\mathscr{I}_{2}\right\|_{H^{1 / 2}(\Gamma)} & \leq(d+1) ! \sum_{k \geq 1} \frac{1}{k^{\frac{2}{d}(d+2)}}\left\|\partial_{\nu} \varphi_{k}^{1}-\partial_{\nu} \varphi_{k}^{2}\right\|_{H^{1 / 2}(\Gamma)}\|f\|_{L^{2}(\Gamma)} \\
& \leq C\left\|\partial_{\nu} \varphi^{1}-\partial_{\nu} \varphi^{2}\right\|_{\ell_{\mathbf{w}}^{1}\left(H^{1 / 2}(\Gamma)\right)}\|f\|_{L^{2}(\Gamma)} .
\end{aligned}
$$

Using the same argument, we obtain

$$
\left\|\mathscr{I}_{3}\right\|_{H^{1 / 2}(\Gamma)} \leq C\left\|\partial_{\nu} \varphi^{1}-\partial_{\nu} \varphi^{2}\right\|_{\ell_{\mathbf{w}}^{1}\left(H^{1 / 2}(\Gamma)\right)}\|f\|_{L^{2}(\Gamma)} .
$$

Combining (2.10), (2.11) and (2.12), we have the desired inequality given by (2.4).

Now, we will prove the second inequality given by (2.5). So to obtain the remaining cases $(j<d+1)$, we write Taylor's formula with remainder, we may write, for $1 \leq j \leq d$.

$$
\mathscr{P}^{(j)}(0)=\sum_{h=j}^{d} \frac{\mathscr{P}^{(h)}(\lambda)}{(h-j) !}(-\lambda)^{h-j}+\int_{\lambda}^{0} \frac{(-\tau)^{d-j}}{(d-j) !} \mathscr{P}^{(d+1)}(\tau) d \tau .
$$

Using Lemma 2.2, we have

$$
\left\|\mathscr{P}^{(h)}(\lambda)\right\|_{\frac{3}{2}, s} \leq C|\lambda|^{-h+\frac{1}{4}(1+2 s)} .
$$

Then

$$
\begin{aligned}
\left\|\mathscr{P}^{(h)}(0)\right\|_{\frac{3}{2}, s} & \leq C\left(\sum_{h=j}^{d}|\lambda|^{h-j}|\lambda|^{-h+\frac{1}{4}(1+s)}+|\lambda|^{d-j+1}\left\|\mathscr{P}^{(d+1)}\right\|_{\frac{3}{2}, s}\right) \\
& \leq C\left(\sum_{h=j}^{d}|\lambda|^{-j+\frac{1}{4}(1+2 s)}+|\lambda|^{d-j+1}\left\|\mathscr{P}^{(d+1)}\right\|_{\frac{3}{2}, s}\right) .
\end{aligned}
$$

Using (2.4), we obtain

$$
\left\|\mathscr{P}^{(h)}(0)\right\|_{\frac{3}{2}, s} \leq C\left(\sum_{h=j}^{d}|\lambda|^{-j+\frac{1}{4}(1+2 s)}+|\lambda|^{d-j+1} \delta\right) .
$$

We suppose that $|\lambda| \geq 1$, so it easy to see that $|\lambda|^{-j+\frac{1}{2}} \leq 1$ and $|\lambda|^{-j} \leq 1$ for $j \geq 1$. Then we have

$$
\left\|\mathscr{P}^{(h)}(0)\right\|_{\frac{3}{2}, s} \leq C\left(|\lambda|^{-\frac{1}{4}(1-2 s)}+|\lambda|^{d+1} \delta\right) \text { for }|\lambda| \geq 1 .
$$

For $|\lambda|=\delta^{-\frac{4}{4 d+5-2 s}}$ and for $\theta=1-\frac{4 d-4}{4 d+5-2 s}$.

This completes the proof. 
Lemma 2.5 There exists $C>0$ such that the following estimate is holds

$$
\left\|\mathscr{R}^{\sharp}\right\|_{s} \leq C \delta .
$$

We remind that $\|\cdot\|_{s}$ is the norm in $\mathcal{L}\left(H^{2 d+4}\left(0, T ; H^{3 / 2}(\Gamma)\right), L^{2}\left((0, T) ; H^{s}(\Gamma)\right)\right)$.

Proof. We denote $f_{j}=\left(\frac{-\partial^{2}}{\partial s^{2}}\right)^{j} f$, since $A_{1}=A_{2}$ on $\Gamma$, then

$$
\begin{aligned}
\mathscr{R}^{\sharp}(f)= & \sum_{k \geq 0}\left(\lambda_{k}^{1}\right)^{-d-5 / 2} \partial_{\nu} \varphi_{k}^{1} \int_{0}^{t} \sin \sqrt{\lambda_{k}^{1}}(t-s)\left\langle f_{d+2}(s, .), \partial_{\nu} \varphi_{k}^{1}(.)\right\rangle d s \\
& -\sum_{k \geq 0}\left(\lambda_{k}^{2}\right)^{-d-5 / 2} \partial_{\nu} \varphi_{k}^{2} \int_{0}^{t} \sin \sqrt{\lambda_{k}^{2}}(t-s)\left\langle f_{d+2}(s, .), \partial_{\nu} \varphi_{k}^{2}(.)\right\rangle d s .
\end{aligned}
$$

We split $\mathscr{R}^{\sharp}(f)$ into three terms $\mathscr{R}^{\sharp}(f)=\mathscr{F}_{1}+\mathscr{F}_{2}+\mathscr{F}_{3}$, where

$$
\begin{gathered}
\mathscr{F}_{1}=\sum_{k \geq 0} \partial_{\nu} \varphi_{k}^{1} \int_{0}^{t}\left[\left(\frac{\sin \sqrt{\lambda_{k}^{1}}(t-s)}{\left(\lambda_{k}^{1}\right)^{d+5 / 2}}-\frac{\sin \sqrt{\lambda_{k}^{2}}(t-s)}{\left(\lambda_{k}^{2}\right)^{d+5 / 2}}\right)\left\langle f_{d+2}(s, .), \partial_{\nu} \varphi_{k}^{1}(.)\right\rangle\right] d s \\
\mathscr{F}_{2}=\sum_{k \geq 0} \frac{1}{\left(\lambda_{k}^{2}\right)^{d+5 / 2}}\left(\partial_{\nu} \varphi_{k}^{1}-\partial_{\nu} \varphi_{k}^{2}\right) \int_{0}^{t} \sin \sqrt{\lambda_{k}^{2}}(t-s)\left\langle f_{d+2}(s, .), \partial_{\nu} \varphi_{k}^{1}(.)\right\rangle d s \\
\mathscr{F}_{3}=\sum_{k \geq 0} \frac{1}{\left(\lambda_{k}^{2}\right)^{d+5 / 2}} \frac{\partial \varphi_{k}^{2}}{\partial \nu} \cdot \int_{0}^{t} \sin \sqrt{\lambda_{k}^{2}}(t-s)\left\langle f_{d+2}(s, .),\left(\partial_{\nu} \varphi_{k}^{1}(.)-\partial_{\nu} \varphi_{k}^{2}(.)\right)\right\rangle d s .
\end{gathered}
$$

So we have the following estimates

$$
\left\|\mathscr{F}_{1}\right\|_{H^{1 / 2}(\Gamma)} \leq \sum_{k \geq 0}\left\|\frac{\partial \varphi_{k}^{1}}{\partial \nu}\right\|_{H^{1 / 2}(\Gamma)}^{2} \int_{0}^{t}\left|\frac{\sin \sqrt{\lambda_{k}^{1}}(t-s)}{\left(\lambda_{k}^{1}\right)^{d+5 / 2}}-\frac{\sin \sqrt{\lambda_{k}^{2}}(t-s)}{\left(\lambda_{k}^{2}\right)^{d+5 / 2}}\right|\left\|f_{d+2}(s, .)\right\|_{L^{2}(\Gamma)} d s .
$$

On the other hand we have

$$
\begin{aligned}
\left|\frac{\sin \sqrt{\lambda_{k}^{1}}(t-s)}{\left(\lambda_{k}^{1}\right)^{d+5 / 2}}-\frac{\sin \sqrt{\lambda_{k}^{2}}(t-s)}{\left(\lambda_{k}^{2}\right)^{d+5 / 2}}\right| & \leq \max _{k \geq 1}\left(\frac{1}{\left(\lambda_{k}^{1}\right)^{d+3}}, \frac{1}{\left(\lambda_{k}^{2}\right)^{d+3}}\right)\left|\lambda_{k}^{1}-\lambda_{k}^{2}\right| \\
& \leq \frac{1}{k^{\frac{2}{d}(d+3)}}\left|\lambda_{k}^{1}-\lambda_{k}^{2}\right| .
\end{aligned}
$$

So we have

$$
\left\|\mathscr{F}_{1}\right\|_{H^{1 / 2}(\Gamma)} \leq \sum_{k \geq 0}\left\|\frac{\partial \varphi_{k}^{1}}{\partial \nu}\right\|_{H^{1 / 2}(\Gamma)}^{2} \frac{1}{k^{\frac{2}{d}(d+3)}}\left|\lambda_{k}^{1}-\lambda_{k}^{2}\right| \int_{0}^{t}\left\|f_{d+2}(s, .)\right\|_{L^{2}(\Gamma)} d s .
$$


When we use (2.8), we obtain

$$
\begin{aligned}
\left\|\mathscr{F}_{1}\right\|_{H^{1 / 2}(\Gamma)} & \leq \sum_{k \geq 0} \frac{1}{k^{\frac{2}{d}(d+3)}}\left|\lambda_{k}^{1}-\lambda_{k}^{2}\right| \int_{0}^{t}\left\|f_{d+2}(s, .)\right\|_{L^{2}(\Gamma)} d s \\
& \leq C\left\|\lambda^{1}-\lambda^{2}\right\|_{\ell_{\mathbf{w}}^{1}(\mathbb{C})}\|f\|_{H^{2 d+4}\left(0, T ; H^{1 / 2}(\Gamma)\right)} .
\end{aligned}
$$

Furthermore

$$
\begin{aligned}
\left\|\mathscr{F}_{2}\right\|_{H^{1 / 2}(\Gamma)} & \leq \sum_{k \geq 0} \frac{1}{k^{\frac{2}{d}(d+5 / 2)}}\left|\frac{\partial \varphi_{k}^{1}}{\partial \nu}-\frac{\partial \varphi_{k}^{2}}{\partial \nu}\right|\left\|\frac{\partial \varphi_{k}^{1}}{\partial \nu}\right\|_{H^{1 / 2}(\Gamma)} \int_{0}^{t}\left\|f_{d+2}(s, .)\right\|_{L^{2}(\Gamma)} d s \\
& \leq \sum_{k \geq 0} \frac{1}{k^{\frac{2}{d}(d+5 / 2)}}\left|\frac{\partial \varphi_{k}^{1}}{\partial \nu}-\frac{\partial \varphi_{k}^{2}}{\partial \nu}\right| \int_{0}^{t}\left\|f_{d+2}(s, .)\right\|_{L^{2}(\Gamma)} d s \\
& \leq\left\|\partial_{\nu} \varphi^{1}-\partial_{\nu} \varphi^{2}\right\|_{\ell_{\mathbf{w}}^{1}\left(H^{1 / 2}(\Gamma)\right)}\|f\|_{H^{2 d+4}\left(0, T ; H^{1 / 2}(\Gamma)\right)} .
\end{aligned}
$$

Using the same calculus we obtain

$$
\left\|\mathscr{F}_{3}\right\|_{H^{1 / 2}(\Gamma)} \leq\left\|\partial_{\nu} \varphi^{1}-\partial_{\nu} \varphi^{2}\right\|_{\ell_{\mathbf{w}}^{1}\left(H^{1 / 2}(\Gamma)\right)}\|f\|_{H^{2 d+4}\left(0, T ; H^{1 / 2}(\Gamma)\right)} .
$$

As a conclusion we found the following estimate

$$
\|\mathscr{R} \sharp\|_{s} \leq C \delta .
$$

This completes the proof of the Lemma.

\subsection{End of the proof of Theorem 1}

Using the result of Lemma 2.4, we obtain the following estimate for any $\varphi \in H^{3 / 2}(\Gamma)$

$$
\left\|\left[\left(\frac{d}{d \lambda}\right)^{j}\left(\Pi_{A_{1}, q_{1}}(\lambda)-\Pi_{A_{2}, q_{2}}(\lambda)\right)\right]_{\lambda=0} \varphi\right\|_{H^{s}(\Gamma)} \leq \delta^{\theta}\|\varphi\|_{H^{3 / 2}(\Gamma)} .
$$

We rely on the decomposition given (2.3), it follows from (2.19) that for any $\psi \in H^{2 d+4}\left(0, T ; H^{3 / 2}(\Gamma)\right)$

$$
\left\|\left[\left(\frac{d}{d \lambda}\right)^{j}\left(\Pi_{A_{1}, q_{1}}(\lambda)-\Pi_{A_{2}, q_{2}}(\lambda)\right)\right]_{\lambda=0} \psi\right\|_{L^{2}\left(0, T ; H^{s}(\Gamma)\right)} \leq \delta^{\theta}\|\psi\|_{H^{2 d+4}\left(0, T ; H^{3 / 2}(\Gamma)\right)} .
$$

Using the estimation given above and the result of Lemma 2.5, we have

$$
\left\|\Lambda_{A_{1}, q_{1}}^{\sharp}-\Lambda_{A_{2}, q_{2}}^{\sharp}\right\|_{s} \leq C \delta^{\theta},
$$

provided that $\delta$ is sufficiently small.

Combining this estimate with (1.13), we obtain the result of Theorem 1. 


\section{Stability estimate for the inverse problem from the D-to-N map}

In this section, we are interested in the proof of the Theorems 2 and 3. For this, we will prove the two following estimates which are given in the Theorem 2

$$
\begin{gathered}
\left\|d \alpha_{A_{1}}-d \alpha_{A_{2}}\right\|_{L^{\infty}(\Omega)} \leq C\left\|\Lambda_{A_{1}, q_{1}}-\Lambda_{A_{2}, q_{2}}\right\|^{\mu^{\prime}} . \\
\left\|q_{1}-q_{2}\right\|_{H^{-1}(\Omega)} \leq C\left\|\Lambda_{A_{1}, q_{1}}-\Lambda_{A_{2}, q_{2}}\right\|^{\mu^{\prime}} .
\end{gathered}
$$

We have the same estimates when we replace $\left\|\Lambda_{A_{1}, q_{1}}-\Lambda_{A_{2}, q_{2}}\right\|$ by $\left\|\Lambda_{A_{1}, q_{1}}^{\sharp}-\Lambda_{A_{2}, q_{2}}^{\sharp}\right\|$ and this is the result of Thorem 3 .

To prove the first inequality, we repeat the same idea in Bellassoued and Ben Joud [3] but the difference between the two works is that in [3], we consider only the wave operator in the magnetic field and in this work we added an electric potential. That does not modify neither the Construction of the geometrical optic solutions nor the proofs, we just add in the term $V$, given in the lemma 3.1 in [3], the term $\left(q_{1}-\right.$ $\left.q_{2}\right) u_{2}$ and the following estimate

$$
\left|\int_{Q} V(x) u_{2}(t, x) \cdot \bar{v}(t, x) d x d t\right| \leq C \mathcal{N}_{\omega}\left(\phi_{2}\right) \mathcal{N}_{\omega}\left(\phi_{1}\right)
$$

is still true. The norm $\mathcal{N}_{\omega}(\phi)$ is given after that.

The estimate for the electric potentials is slightly more involved and the complications would arise when one tries to establish the estimate for the electric potential (lower order) in the presence of the magnetic field (higher order). To remedy to this difficulty, we first show by using the Hodge decomposition that the $d$ operator on differential forms in some sense "bounded invertible" when restricted to right subspaces. Then we will combine this fact with the estimate we have for $d \alpha_{A_{1}}-d \alpha_{A_{2}}$ to obtain the estimate for electric potentials. The rest of this paper is devoted to prove an estimate of the electric potential.

\subsection{Construction of geometrical optics solutions}

The following result concerning the existence of the geometrical optics solutions for the magnetic wave equation will be important to prove our main result. From the hypothesis there exists $\varrho>0$ such that $T>T-4 \varrho>D_{\Omega}$ and $\bar{\Omega}$ lies in the ball $B\left(x_{0}, \frac{T}{2}-2 \varrho\right)$. We may assume without loss of generality that $x_{0}$ is the origin of $\mathbb{R}^{d}$, and let

$$
\mathfrak{D}_{\varrho}=B\left(0, \frac{T}{2}\right) \backslash \bar{B}\left(0, \frac{T}{2}-2 \varrho\right)=\left\{x \in \mathbb{R}^{d}: \frac{T}{2}-2 \varrho<|x|<\frac{T}{2}\right\} .
$$

Let $\phi \in \mathcal{C}_{0}^{\infty}\left(\mathbb{R}^{d}\right)$ such that

$$
\operatorname{supp}(\phi) \subset \mathfrak{D}_{\varrho} .
$$

Thus we have

$$
\operatorname{supp} \phi \cap \Omega=\emptyset, \quad(\operatorname{supp} \phi \pm T \omega) \cap \Omega=\emptyset, \quad \forall \omega \in \mathbb{S}^{d-1} .
$$

Moreover the function $\Phi(t, x)=\phi(x+t \omega)$ solves in $\mathbb{R} \times \mathbb{R}^{d}$ the transport equation

$$
\left(\partial_{t}-\omega \cdot \nabla\right) \Phi(t, x)=0 .
$$

Finally, for $\omega \in \mathbb{S}^{d-1}$ we set

$$
\mathcal{H}_{\omega}^{2}\left(\mathfrak{D}_{\varrho}\right)=\left\{\phi \in H^{2}\left(\mathbb{R}^{d}\right), \quad \omega \cdot \nabla \phi \in H^{2}\left(\mathbb{R}^{d}\right), \quad \text { and } \quad \operatorname{supp}(\phi) \subset \mathfrak{D}_{\varrho}\right\}
$$


and

$$
\mathcal{N}_{\omega}(\phi)=\|\phi\|_{H^{2}\left(\mathbb{R}^{d}\right)}+\|\omega \cdot \nabla \phi\|_{H^{2}\left(\mathbb{R}^{d}\right)} .
$$

Now we recall the following Lemma which is proved in [3].

Lemma 3.1 Let $\omega \in \mathbb{S}^{d-1}, \phi \in \mathcal{H}_{\omega}^{2}\left(\mathfrak{D}_{\varrho}\right), A \in W^{3, \infty}\left(\Omega ; \mathbb{R}^{d}\right), q \in L^{\infty}(\Omega)$ and $\tau>0$, then

$$
\left(\partial_{t}^{2}-\Delta_{A}+q\right) u(t, x)=0, \quad(t, x) \in Q=(0, T) \times \Omega
$$

has a solution of the form

$$
u(t, x)=\phi(x+t \omega) b(t, x) e^{i \tau(x \cdot \omega+t)}+\psi_{\tau}(t, x),
$$

where

$$
b(t, x)=\exp \left(i \int_{0}^{t} \omega \cdot A(x+s \omega) d s\right)
$$

and $\psi_{\tau}(t, x)$ satisfies

$$
\psi_{\tau}(t, x)=0, \quad \text { for all } \quad(t, x) \in \Sigma
$$

and

$$
\psi_{\tau}(\theta, x)=0, \quad x \in \Omega, \quad \theta=0 \text { or } T .
$$

Moreover

$$
\tau\left\|\psi_{\tau}\right\|_{L^{2}(Q)}+\left\|\nabla \psi_{\tau}\right\|_{L^{2}(Q)} \leq C \mathcal{N}_{\omega}(\phi)
$$

where $C$ is a constant depending only on $\Omega, T, d$ and $\|A\|_{W^{3, \infty}\left(\Omega ; \mathbb{R}^{d}\right)}$.

\subsection{Hodge decomposition}

We consider $(\Omega, \Gamma)$ to be a Riemannian manifold with boundary and denote by $F^{k}(\Omega)$ to be set of $k$ forms on $\Omega$ and $W^{m, p} F^{k}(\Omega)$ to be its $W^{m, p}$ closure. Set

$$
\mathcal{E}^{k}(\Omega):=\left\{d \alpha ; \alpha \in H_{D}^{1} F^{k-1}(\Omega)\right\}, \mathcal{C}^{k}(\Omega):=\left\{d \alpha ; \alpha \in H_{N}^{1} F^{k-1}(\Omega)\right\},
$$

where $H_{D}^{1} F^{k}(\Omega)$ and $H_{N}^{1} F^{k}(\Omega)$ are the set of $H^{1} k$-forms with homogenous Dirichlet and Neumann boundary trace, respectively. Furthermore, we denote by $\mathcal{H}^{k}(\Omega)$ to be the $L^{2}$ closed of the space of harmonic $k$-forms. The corresponding subspaces in $W^{m, p} F^{k}(\Omega)$ are denoted by

$$
W^{m, p} \mathcal{E}^{k}(\Omega):=\mathcal{E}^{k}(\Omega) \cap W^{m, p} F^{k}(\Omega), W^{m, p} \mathcal{C}^{k}(\Omega):=\mathcal{C}^{k}(\Omega) \cap W^{m, p} F^{k}(\Omega)
$$

and

$$
W^{m, p} \mathcal{H}^{k}(\Omega):=\mathcal{H}^{k}(\Omega) \cap W^{m, p} F^{k}(\Omega) .
$$

Finally, we denote

$$
W_{D}^{m, p}\left(\Omega ; \mathbb{R}^{n}\right)=\left\{A \in W^{m, p}\left(\Omega ; \mathbb{R}^{n}\right) ; A . \widehat{\tau}=0, \forall \widehat{\tau} \in T_{x} \Gamma, x \in \Gamma\right\}
$$

and

$$
X_{0}=\left(W^{m, p} \mathcal{C}^{1}(\Omega) \oplus W^{m, p} \mathcal{H}^{1}(\Omega)\right) \cap W_{D}^{m, p}\left(\Omega ; \mathbb{R}^{n}\right) .
$$


In the classical form the Hodge decomposition theorem states that if $\Omega$ is a smooth subdomain of smooth compact Riemannian manifold, then any smooth differential form $A$ in $\Omega$ can be decomposed as

$$
A=d \alpha+\delta \beta+\kappa
$$

where $\alpha, \beta, \kappa$ are smooth differential forms in $\Omega$, two of which have a vanishing normal or tangential component on $\Omega$ and so that $d \kappa=\delta \kappa=0$. Here $d$ is the exterior differential operator and $\delta$ is the codifferential.

If $A \in W^{m, p} F^{1}$ and $\Omega$ has a smooth boundary, then on has Hodge decomposition given by

$$
A=d \alpha+\delta \beta+\kappa \text { and } \alpha \in W_{D}^{m+1, p}(\Omega, \mathbb{R}), \delta \beta \in W^{m, p} \mathcal{C}^{1}(\Omega) \text { and } \kappa \in W^{m, p} \mathcal{H}^{1}(\Omega) .
$$

Further, the three summands in (3.9) are uniquely determined and mutually orthogonal with respect to the natural $L^{2}$ inner product.

Based on the Hodge decomposition, Tzou showed in [34], that for $p \geq 2$ and $m \geq 1$

$$
d: X_{0} \longrightarrow W^{m-1, p} \mathcal{E}^{2}(\Omega) \text { has a bounded inverse. }
$$

This statement means that for all $A \in W^{m-1, p} \mathcal{E}^{2}(\Omega)$ we have $\|A\|_{W^{m, p}\left(\Omega, \mathbb{R}^{n}\right)} \leq C\|d A\|_{W^{m-1, p}\left(\Omega, \mathbb{R}^{n}\right)}$. We would like to apply this result to the vector field $\left(A_{1}-A_{2}\right)$ which may not be in $X_{0}$. For that, pick $p>n$ and apply the Hodge decomposition to $\left(A_{1}-A_{2}\right)$ in the space $W^{3, \infty}\left(\Omega ; \mathbb{R}^{n}\right)$ to get $A_{1}-A_{2}=$ $d \alpha+\delta \beta+\kappa$, where $\alpha \in W^{4, \infty}(\Omega) \cap H_{0}^{1}(\Omega)$. Define $A_{1}^{\prime}=A_{1}-\frac{d \alpha}{2}, A_{2}^{\prime}=A_{2}+\frac{d \alpha}{2}$ so that $A_{1}^{\prime}-A_{2}^{\prime} \in W^{3, \infty} \mathcal{C}^{1}(\Omega) \oplus W^{3, \infty} \mathcal{H}^{1}(\Omega)$. Since we have already assumed that $A_{1}-A_{2}=0$ in $\Gamma$, it is easy to show that it has no tangential component at the boundary and $\alpha \in H_{0}^{1}(\Omega)$, we conclude that $A_{1}^{\prime}-A_{2}^{\prime}$ has no tangential component. This means that

$$
A_{1}^{\prime}-A_{2}^{\prime} \in W^{3, \infty} \mathcal{C}^{1}(\Omega) \oplus W^{3, \infty} \mathcal{H}^{1}(\Omega) \cap H_{D}^{1}\left(\Omega ; \mathbb{R}^{n}\right) .
$$

So by the Lemma 6.2 from [34]

$$
\left\|A_{1}^{\prime}-A_{2}^{\prime}\right\|_{W^{3, \infty}(\Omega)} \leq C\left\|d \alpha_{A_{1}^{\prime}}-d \alpha_{A_{2}^{\prime}}\right\|_{L^{\infty}(\Omega)}=\left\|d \alpha_{A_{1}}-d \alpha_{A_{2}}\right\|_{L^{\infty}(\Omega)} .
$$

Gauge equivalence then implies that $\Lambda_{A_{j}^{\prime}, q_{j}}=\Lambda_{A_{j}, q_{j}}$ for $j=1,2$.

Remark 2 With this choice of $A_{1}^{\prime}$ and $A_{2}^{\prime}$, we remake that div $\left(A_{1}^{\prime}-A_{2}^{\prime}\right)=0$. This come from the fact that on the 1-form div $=* d *$, so using this definition, the Lemma 4.1 in Ben Joud [7] and the fact that $\kappa$ is an harmonic form we obtain

$$
\operatorname{div}\left(A_{1}^{\prime}-A_{2}^{\prime}\right)=\operatorname{div}(\delta \beta+\kappa)=* d * \delta \beta+* d * \kappa=-\delta \delta \beta-\delta \kappa=0 .
$$

We will from now, make the same work again with the magnetic field defined above. In this case, with this special forms of $A_{1}^{\prime}$ and $A_{2}^{\prime}$, there is a condition change given by $A_{1}^{\prime}-A_{2}^{\prime}$ is not vanish in $\Gamma$ but it has not tangential component.

As before, we set

$$
A^{\prime}(x)=\left(A_{1}^{\prime}-A_{2}^{\prime}\right)(x)
$$

and

$$
V^{\prime}(x)=-i \operatorname{div}\left(A^{\prime}\right)+\left(A_{2}^{\prime 2}-A_{1}^{\prime 2}\right)(x)=\left(A_{2}^{\prime 2}-A_{1}^{\prime 2}\right)(x)=\left(A_{2}^{\prime}-A_{1}^{\prime}\right)\left(A_{2}^{\prime}+A_{1}^{\prime}\right) .
$$

Recall that since $\left(A_{1}^{\prime}-A_{2}^{\prime}\right)=0$ and $\left(q_{1}-q_{2}\right)=0$ on $\Gamma$, we can extend $A^{\prime}$ to a $H^{1}\left(\mathbb{R}^{d}\right)$ vector field and $q$ to $L^{\infty}\left(\mathbb{R}^{d}\right)$ by defining it to be zero outside of $\Omega$ and we will refer to the extension as $A^{\prime}$ and $q$. We can extend $V$ to $L^{\infty}\left(\mathbb{R}^{d}\right)$ function by defining it to be zero outside of $\Omega$ and we will refer to the extension as $V$. 


\subsection{Preliminaries estimates}

In this subsection, we complete the proof of Theorem 2. We are going to use the geometrical optics solutions and the $x$-ray transform of the difference of two magnetic potentials.

As before, we let $\omega \in \mathbb{S}^{d-1}$ and $A_{l} \in W^{3, \infty}\left(\Omega ; \mathbb{R}^{d}\right), q_{l} \in L^{\infty}\left(\Omega ; \mathbb{R}^{d}\right)$ such that $\left\|A_{l}\right\|_{W^{3, \infty}} \leq M$ and $\left\|q_{l}\right\|_{L^{\infty}} \leq M$ for $l=1,2$. We set $A^{\prime}(x)=\left(A_{2}^{\prime}-A_{1}^{\prime}\right)(x), q(x)=\left(q_{2}-q_{1}\right)(x)$ and

$$
b(t, x)=\left(b_{2} \bar{b}_{1}\right)(t, x)=\exp \left(i \int_{0}^{t} \omega \cdot A^{\prime}(x+s \omega) d s\right) .
$$

Recall that since $\left(A_{1}^{\prime}-A_{2}^{\prime}\right)=0$ and $q_{1}-q_{2}=0$ on $\Gamma$, we can extend $A^{\prime}$ to a $H^{1}\left(\mathbb{R}^{d}\right)$ vector field and $q$ to $L^{\infty}\left(\mathbb{R}^{d}\right)$ by defining it to be zero outside of $\Omega$ and we will refer to the extention as $A^{\prime}$ and $q$. With this extention we have that $d \alpha_{A}^{\prime}$ is an $L^{2}\left(\mathbb{R}^{d}\right)$ function supported only in $\Omega$.

Lemma 3.2 There exists $C>0$ such that for any $\omega \in \mathbb{S}^{d-1}$ and $\phi_{1}, \phi_{2} \in \mathcal{H}_{\omega}^{2}\left(\mathfrak{D}_{\varrho}\right)$ the following estimates holds true:

$$
\begin{aligned}
\left|\int_{0}^{T} \int_{\mathbb{R}^{d}} q(x+t \omega) b(t, x) d x d t\right| \leq & C \tau^{3}\left\|\phi_{1}\right\|_{H^{1}\left(\mathbb{R}^{d}\right)}\left\|\phi_{2}\right\|_{H^{2}\left(\mathbb{R}^{d}\right)}\left\|\Lambda_{A_{1}, q_{1}}-\Lambda_{A_{2}, q_{2}}\right\| \\
& +\frac{C}{\tau} \mathcal{N}_{\omega}\left(\phi_{1}\right) \mathcal{N}_{\omega}\left(\phi_{2}\right)+C \tau\left\|A^{\prime}\right\|_{L^{\infty}} \mathcal{N}_{\omega}\left(\phi_{1}\right) \mathcal{N}_{\omega}\left(\phi_{2}\right)
\end{aligned}
$$

for any sufficiently large $\tau>0$.

Proof . For $\tau$ sufficiently large, Lemma 3.1 guarantees the existence of the geometrical optics solutions $u_{2}$ to

$$
\left(\partial_{t}^{2}-\Delta_{A_{2}^{\prime}}+q_{2}\right) u(t, x)=0 \quad \text { in } Q, \quad u(0, \cdot)=\partial_{t} u(0, \cdot)=0 \quad \text { in } \Omega
$$

in the form

$$
u_{2}(t, x)=\phi_{2}(x+t \omega) b_{2}(t, x) e^{i \tau(x . \omega+t)}+\psi_{2, \tau}(t, x),
$$

corresponding to the magnetic potential $A_{2}$ and $\phi_{2}$, where $\psi_{2, \tau}$ satisfies

$$
\tau\left\|\psi_{2, \tau}\right\|_{L^{2}(Q)}+\left\|\nabla \psi_{2, \tau}\right\|_{L^{2}(Q)} \leq C \mathcal{N}_{\omega}\left(\phi_{2}\right)
$$

and

$$
u_{2} \in \mathcal{C}^{1}\left(0, T ; L^{2}(\Omega)\right) \cap \mathcal{C}\left(0, T ; H^{1}(\Omega)\right) .
$$

We denote $u_{1}$, the solution of

$$
\begin{cases}\left(\partial_{t}^{2}-\Delta_{A_{1}^{\prime}}+q_{1}\right) u_{1}=0 & (t, x) \in Q, \\ u_{1}(0, x)=\partial_{t} u_{1}(0, x)=0 & x \in \Omega, \\ u_{1}(t, x)=u_{2}(t, x):=f_{\tau}(t, x) & (t, x) \in \Sigma .\end{cases}
$$

Defining $u=u_{1}-u_{2}$, one gets

$$
\begin{cases}\left(\partial_{t}^{2} u-\Delta_{A_{1}^{\prime}}+q_{1}\right) u(t, x)=2 i A^{\prime} \cdot \nabla u_{2}(t, x)+V^{\prime}(x) u_{2}(t, x)+q(x) & (t, x) \in Q, \\ u(0, x)=\partial_{t} u(0, x)=0 & x \in \Omega, \\ u(t, x)=0 & (t, x) \in \Sigma .\end{cases}
$$


where $V^{\prime}(x)$ is given in (3.12).

Therefore, we have constructed the special solution $v \in \mathcal{C}^{1}\left(0, T ; L^{2}(\Omega)\right) \cap \mathcal{C}\left(0, T ; H^{1}(\Omega)\right)$ to the backward magnetic wave equation

$$
\left(\partial_{t}^{2}-\Delta_{A_{1}^{\prime}}+q_{1}\right) v(t, x)=0, \quad(t, x) \in Q, \quad v(T, x)=\partial_{t} v(T, x)=0, \quad x \in \Omega,
$$

having the form

$$
v(t, x)=\phi_{1}(x+t \omega) b_{1}(t, x) e^{i \tau(x \cdot \omega+t)}+\psi_{1, \tau}(t, x),
$$

corresponding to the magnetic and electric potential $A_{1}^{\prime}, q_{1}$ and $\phi_{1}$, where $\psi_{1, \tau}$ satisfies

$$
\tau\left\|\psi_{1, \tau}\right\|_{L^{2}(Q)}+\left\|\nabla \psi_{1, \tau}\right\|_{L^{2}(Q)} \leq C \mathcal{N}_{\omega}\left(\phi_{1}\right)
$$

Integrating by parts and using the Green's formula, we obtain

$$
\begin{aligned}
& \int_{Q}\left(\partial_{t}^{2}-\Delta_{A_{1}^{\prime}}+q_{1}\right) u(t, x) \cdot \bar{v}(t, x) d x d t=\int_{Q} 2 i A^{\prime} \cdot \nabla u_{2}(t, x) \cdot \bar{v}(t, x) d x d t \\
& +\int_{Q} V^{\prime}(x) u_{2}(t, x) \cdot \bar{v}(t, x) d x d t+\int_{Q} q(x) u_{2}(t, x) \cdot \bar{v}(t, x) d x d t \\
= & -\int_{\Sigma}\left(\partial_{\nu}+i A_{1}^{\prime} \cdot \nu\right) u(t, x) \bar{v}(t, x) d \sigma_{x} d t .
\end{aligned}
$$

Combining (3.8) with (3.5), we obtain

$$
\begin{aligned}
& \int_{Q} q^{\prime}(x) u_{2}(t, x) \cdot \bar{v}(t, x) d x d t=-\int_{Q} 2 i A^{\prime} \cdot \nabla u_{2}(t, x) \cdot \bar{v}(t, x) d x d t \\
& -\int_{Q} V^{\prime}(x) u_{2}(t, x) \cdot \bar{v}(t, x) d x d t-\int_{\Sigma}\left(\Lambda_{A_{1}, q_{1}}-\Lambda_{A_{2}, q_{2}}\right)\left(f_{\tau}\right)(t, x) \bar{g}_{\tau}(t, x) d \sigma_{x} d t
\end{aligned}
$$

where

$$
g_{\tau}(t, x)=\phi_{1}(x+t \omega) b_{1}(t, x) e^{i \tau(x \cdot \omega+t)}, \quad(t, x) \in \Sigma .
$$

It follows from (3.6) and (3.3) that

$$
\int_{Q} q(x) u_{2}(t, x) \cdot \bar{v}(t, x) d x d t=-\int_{Q} q(x)\left(\phi_{2} \bar{\phi}_{1}\right)(x+t \omega)\left(b_{2} \bar{b}_{1}\right)(t, x) d x d t+\mathcal{I}_{\tau} .
$$

By using (3.7) and (3.4) we obtain

$$
\left|\mathcal{I}_{\tau}\right| \leq \frac{C}{\tau} \mathcal{N}_{\omega}\left(\phi_{2}\right) \mathcal{N}_{\omega}\left(\phi_{1}\right)
$$

Consequently, by (3.11), (3.10) and (3.9), we obtain

$$
\begin{aligned}
& \left|\int_{Q} q(x)\left(\phi_{2} \bar{\phi}_{1}\right)(x+t \omega)\left(b_{2} \bar{b}_{1}\right)(t, x) d x d t\right| \leq\left|\int_{Q} 2 i A^{\prime} \cdot \nabla u_{2}(t, x) \cdot \bar{v}(t, x) d x d t\right| \\
& +C\left|\int_{Q} V(x) u_{2} \cdot \bar{v} d x d t\right|+C\left|\int_{\Sigma}\left(\Lambda_{A_{1}, q_{1}}-\Lambda_{A_{2}, q_{2}}\right)\left(f_{\tau}\right)(t, x) \bar{g}_{\tau}(t, x) d \sigma_{x} d t\right|+\frac{C}{\tau} \mathcal{N}_{\omega}\left(\phi_{2}\right) \mathcal{N}_{\omega}\left(\phi_{1}\right) .
\end{aligned}
$$

Moreover, by (3.7) and (3.4), one gets

$$
\left|\int_{Q} V(x) u_{2} \cdot \bar{v} d x d t\right|=\left|\int_{Q} A^{\prime}(x)\left(A_{1}+A_{2}\right) u_{2} \cdot \bar{v} d x d t\right| \leq C\left\|A^{\prime}\right\|_{L^{\infty}(\Omega)} \mathcal{N}_{\omega}\left(\phi_{2}\right) \mathcal{N}_{\omega}\left(\phi_{1}\right) .
$$


and

$$
\left|\int_{Q} 2 i A^{\prime} \cdot \nabla u_{2}(t, x) \cdot \bar{v}(t, x) d x d t\right| \leq \tau\left\|A^{\prime}\right\|_{L^{\infty}} \mathcal{N}_{\omega}\left(\phi_{2}\right) \mathcal{N}_{\omega}\left(\phi_{1}\right)
$$

By a trace inequality, we have

$$
\begin{gathered}
\left|\int_{\Sigma}\left(\Lambda_{A_{1}, q_{1}}-\Lambda_{A_{2}, q_{2}}\right)\left(f_{\tau}\right)(t, x) \bar{g}_{\tau}(t, x) d \sigma_{x} d t\right| \leq\left\|\Lambda_{A_{1}, q_{1}}-\Lambda_{A_{2}, q_{2}}\right\|\left\|f_{\tau}\right\|_{H^{1}(\Sigma)}\left\|g_{\tau}\right\|_{L^{2}(\Sigma)} \\
\leq C \tau^{3}\left\|\phi_{1}\right\|_{H^{1}\left(\mathbb{R}^{d}\right)}\left\|\phi_{2}\right\|_{H^{2}\left(\mathbb{R}^{d}\right)}\left\|\Lambda_{A_{1}, q_{1}}-\Lambda_{A_{2}, q_{2}}\right\| .
\end{gathered}
$$

Thus, from (3.12), (3.13) and (3.14), we derive for $\tau$ sufficiently large

$$
\begin{aligned}
& \left|\int_{0}^{T} \int_{\mathbb{R}^{n}} q(x) \phi_{2}(x+t \omega) \bar{\phi}_{1}(x+t \omega) b(t, x) d x d t\right| \leq \tau^{3}\left\|\phi_{1}\right\|_{H^{1}}\left\|\phi_{2}\right\|_{H^{2}}\left\|\Lambda_{A_{1}, q_{1}}-\Lambda_{A_{2}, q_{2}}\right\| \\
& +\tau\left\|A^{\prime}\right\|_{L^{\infty}(\Omega)} \mathcal{N}_{\omega}\left(\phi_{1}\right) \mathcal{N}_{\omega}\left(\phi_{2}\right)+\frac{C}{\tau} \mathcal{N}_{\omega}\left(\phi_{1}\right) \mathcal{N}_{\omega}\left(\phi_{2}\right) .
\end{aligned}
$$

This completes the proof of the lemma.

\subsection{Stability of the ray transform}

The $x$-ray transform $\mathcal{P}$ maps a function in $\mathbb{R}^{d}$ into the set of its line integrals. More precisely, if $\omega \in \mathbb{S}^{d-1}$ and $x \in \mathbb{R}^{d}$,

$$
\mathcal{P}(f)(\omega, x):=\int_{\mathbb{R}} f(x+s \omega) d s,
$$

is the integral of $f$ over the straight line through $x$ with the direction $\omega$. It is easy to see that $\mathcal{P}(f)(\omega, x)$ does not change if $x$ is moved in the direction $\omega$. Therefore we normally restrict $x$ to

$$
\omega^{\perp}=\left\{\theta \in \mathbb{R}^{d} ; \theta \cdot \omega=0\right\} .
$$

Lemma 3.3 Let $f \in L^{1}\left(\mathbb{R}^{d}\right)$ and $\omega \in \mathbb{S}^{d-1}$. Then $\mathcal{P} f(\omega,.) \in L^{1}\left(\omega^{\perp}\right)$ and

$$
\mathcal{F}(\mathcal{P} f(\omega, .))(\xi)=\sqrt{2 \pi} \hat{f}(\xi)
$$

for all $\xi \in \omega^{\perp}$.

Let

$$
\mathfrak{D}_{\varrho}^{+}=\left\{x \in \mathfrak{D}_{\varrho}, x \cdot \omega>0\right\} .
$$

We sum up the result of this section in the following lemma.

Lemma 3.4 There exist constants $C>0$ and $\tau_{0}>0$ such that for all $\omega \in \mathbb{S}^{d-1}$ and $\phi$ satisfying $\operatorname{supp}(\phi) \subset \mathfrak{D}_{\varrho}^{+}$, the following estimate

$$
\begin{gathered}
\left|\int_{\mathbb{R}^{d}} \phi^{2}(x) \mathcal{P}(q)(\omega, x) \exp \left(i \int_{\mathbb{R}} \omega \cdot A^{\prime}(x-s \omega) d s\right) d x\right| \leq \tau^{3}\|\phi\|_{H^{2}}^{2}\left\|\Lambda_{A_{1}, q_{1}}-\Lambda_{A_{2}, q_{2}}\right\| \\
+\frac{C}{\tau} \mathcal{N}_{\omega}(\phi)^{2}+C\left\|A^{\prime}\right\|_{L^{\infty}(\Omega)} \mathcal{N}_{\omega}(\phi)^{2}
\end{gathered}
$$

holds for any $\tau \geq \tau_{0}$ and $j \in\{1, \cdots, d\}$. 
Proof . Let $\phi_{1}, \phi_{2} \in \mathcal{H}_{\omega}^{2}\left(\mathfrak{D}_{\varrho}\right) \operatorname{such}$ that $\operatorname{supp}\left(\phi_{j}\right) \subset \mathfrak{D}_{\varrho}^{+}$, we have

$$
\begin{aligned}
& \int_{0}^{T} \int_{\mathbb{R}^{d}} q(x)\left(\phi_{2} \bar{\phi}_{1}\right)(x+t \omega) b(t, x) d x d t \\
& =\int_{0}^{T} \int_{\mathbb{R}^{d}} q(x)\left(\phi_{2} \bar{\phi}_{1}\right)(x+t \omega) \exp \left(i \int_{0}^{t} \omega \cdot q(x+s \omega) d s\right) d x d t \\
& =\int_{0}^{T} \int_{\mathbb{R}^{d}} q(x-t \omega)\left(\phi_{2} \bar{\phi}_{1}\right)(x) b(t, x-t \omega) d x d t \\
& =\int_{\mathbb{R}^{d}}\left(\phi_{2} \bar{\phi}_{1}\right)(x) \int_{0}^{T} q(x-t \omega) \exp \left(i \int_{0}^{t} \omega \cdot A^{\prime}(x-s \omega) d s\right) d x d t \\
& =\int_{\mathbb{R}^{d}}\left(\phi_{2} \bar{\phi}_{1}\right)(x) \int_{0}^{T} q(x-t \omega) \exp \left(i \int_{0}^{T} \omega \cdot A^{\prime}(x-s \omega) d s\right) d x d t \\
& -\int_{\mathbb{R}^{d}}\left(\phi_{2} \bar{\phi}_{1}\right) \int_{0}^{T} q(x-t \omega)(b(T, x)-b(t, x)) d x .
\end{aligned}
$$

Where $b(t, x)=\exp \left(i \int_{0}^{t} \omega \cdot A^{\prime}(x-s \omega) d s\right)$. For $x$ fixed, we denote

$$
b(t, x)=b_{x}(t) .
$$

Using the Mean Value Theorem in the interval $[t, T]$, there exists a $c_{t} \in[t, T]$ such that

$$
\left|b_{x}(T)-b_{x}(t)\right| \leq C(T-t)\left|\omega \cdot A^{\prime}(x-t \omega)\right| .
$$

Choosing $\phi_{1}$ and $\phi_{2}$ such that $\phi_{2}=\phi=\phi_{1}$, (3.19) yields

$$
\begin{aligned}
& \int_{0}^{T} \int_{\mathbb{R}^{d}} q(x)\left(\phi_{2} \bar{\phi}_{1}\right)(x+t \omega) b(t, x) d x d t \\
& =\int_{0}^{T} \int_{\mathbb{R}^{d}} q(x)\left(\phi_{2} \bar{\phi}_{1}\right)(x+t \omega) b(T, x) d x d t+\mathcal{I}_{1} \\
& =\int_{0}^{T} \int_{\mathbb{R}^{d}} q(x) \phi^{2}(x+t \omega) b(T, x) d x d t+\mathcal{I}_{1}
\end{aligned}
$$

where

$$
\left\|\mathcal{I}_{1}\right\|_{L^{2}} \leq C\left\|A^{\prime}\right\|_{L^{\infty}(\Omega)}\|\phi\|_{L^{2}(\Omega)}^{2} \leq C\left\|A^{\prime}\right\|_{L^{\infty}} \mathcal{N}_{\omega}(\phi)^{2} .
$$

Since the support of $A^{\prime}$ and $q$ are contained in $B(0, T / 2-2 \varrho)$, then for $x \in \mathfrak{D}_{\varrho}^{+}$, we have

and

$$
\int_{0}^{T} \omega \cdot A(x-s \omega) d s=\int_{\mathbb{R}} \omega \cdot A(x-s \omega) d s
$$

$$
\int_{0}^{T} q(x-s \omega) d s=\int_{\mathbb{R}} q(x-s \omega) d s .
$$

Indeed, for $s \geq T$ and $x \in \mathfrak{D}_{\varrho}$ it is easy to see that $(x-s \omega) \notin B(0, T / 2-\varrho)$, so

and

$$
\int_{0}^{T} \omega \cdot A^{\prime}(x-s \omega) d s=\int_{0}^{\infty} \omega \cdot A^{\prime}(x-s \omega) d s
$$

$$
\int_{0}^{T} q(x-s \omega) d s=\int_{0}^{\infty} q(x-s \omega) d s
$$


On the other hand, if $s \leq 0$ and $x \in \mathfrak{D}_{\varrho}^{+}$, we get $|x-s \omega|^{2}=|x|^{2}+s^{2}-2 s x \cdot \omega \geq(T / 2-2 \varrho)^{2}$ and then $A^{\prime}(x-s \omega)=0$ and $q(x-s \omega)=0$. This way, (3.22) is obtained.

Substituting (3.22) into the equation (3.21), we obtain

$$
\begin{gathered}
\int_{0}^{T} \int_{\mathbb{R}^{d}} q(x)\left(\phi_{2} \phi_{1}\right)(x+t \omega) b(t, x) d x d t= \\
-\int_{\mathbb{R}^{d}} \phi^{2}(x) \mathcal{P}(q)(\omega, x) \exp \left(i \int_{\mathbb{R}} \omega \cdot A^{\prime}(x-s \omega) d s\right) d x+\mathcal{I}_{1} .
\end{gathered}
$$

By (3.24), (3.2) and the inequality given above, we conclude that for any $\tau \geq \tau_{0}$, it holds that

$$
\begin{aligned}
\mid \int_{\mathbb{R}^{d}} \phi^{2}(x) \mathcal{P}(q)(\omega, x) \exp ( & \left.i \int_{\mathbb{R}} \omega \cdot A^{\prime}(x-s \omega) d s\right) d x \mid \leq \tau^{3}\|\phi\|_{H^{2}}^{2}\left\|\Lambda_{A_{1}, q_{1}}-\Lambda_{A_{2}, q_{2}}\right\| \\
& +\frac{C}{\tau} \mathcal{N}_{\omega}(\phi) \mathcal{N}_{\omega}(\phi)+C \tau\left\|A^{\prime}\right\|_{L^{\infty}(\Omega)}\|\phi\|_{L^{2}(\Omega)}^{2} .
\end{aligned}
$$

This completes the proof of Lemma 3.4.

\subsection{End of proof of Theorem 2}

We shall use the following notations. For $x_{0} \in \omega^{\perp} \cap B(0, T / 2-\varrho)$ we have $B\left(x_{0}, \varrho / 4\right) \cap \omega^{\perp} \subset$ $B(0, T / 2-\varrho / 2) \cap \omega^{\perp}$. Let

$$
\eta_{x_{0}}^{2}=\left(\frac{T}{2}-\frac{\varrho}{2}\right)^{2}-\left|x_{0}\right|^{2}
$$

we set

$$
x_{1}=x_{0}+\eta_{x_{0}} \omega .
$$

It is not difficult to check that $B\left(x_{1}, \varrho / 2\right) \subset \mathfrak{D}_{\varrho}^{+}$. Now, we will get an estimate of the Fourier transform of $q$.

Lemma 3.5 There exist constants $C>0$ and $\tau_{0}>0$ such that the following estimate

$$
|\widehat{q}(\xi)| \leq C \tau^{3}\langle\xi\rangle^{4}\left\|\Lambda_{A_{1}, q_{1}}-\Lambda_{A_{2}, q_{2}}\right\|+\frac{C}{\tau}\langle\xi\rangle^{4}+C \tau\langle\xi\rangle^{4}\left\|\Lambda_{A_{1}, q_{1}}-\Lambda_{A_{2}, q_{2}}\right\|^{\mu} .
$$

holds for all $\tau \geq \tau_{0}$ and all $\xi \in \mathbb{R}^{d}$.

Proof . We fix $x_{0} \in \omega^{\perp} \cap B(0, T / 2-\varrho)$. Let $\theta \in \mathcal{C}_{0}^{\infty}((0, \varrho / 4))$ such that

$$
\int_{\mathbb{R}} \theta^{2}(t) d t=1
$$

and let $\phi_{0} \in \mathcal{C}_{0}^{\infty}\left(\omega^{\perp} \cap B\left(x_{0}, \varrho / 4\right)\right)$ and $\phi_{0} \geq 0$.

Putting

$$
\phi(y)=\theta\left(y \cdot \omega-\eta_{x_{0}}\right) e^{-\frac{i}{2} y \cdot \xi} \phi_{0}^{1 / 2}(y-(y \cdot \omega) \omega) \exp \left(-\frac{i}{2} \int_{\mathbb{R}} \omega \cdot A(y-s \omega) d s\right) .
$$

Then we have

$$
\operatorname{supp}(\phi) \subset B\left(x_{1}, \varrho / 2\right) \subset \mathfrak{D}_{\varrho}^{+} .
$$


The change of variable $y=x+t \omega \in \omega^{\perp} \oplus \mathbb{R} \omega, d y=d \sigma d t$ yields, after noting that $\xi \in \omega^{\perp}$

$$
\begin{aligned}
& \int_{\mathbb{R}^{d}} \phi^{2}(y) \mathcal{P}(q)(\omega, y) \exp \left(i \int_{\mathbb{R}} \omega \cdot A^{\prime}(y-s \omega) d s\right) d y \\
& =\int_{\mathbb{R}} \int_{\omega^{\perp}} \phi^{2}(x+t \omega) \mathcal{P}(q)(\omega, x+t \omega) \exp \left(i \int_{\mathbb{R}} \omega \cdot A^{\prime}(x-s \omega) d s\right) d \sigma d t \\
& =\int_{\mathbb{R}} \int_{\omega^{\perp}} \theta^{2}(t) e^{-i x \cdot \xi} \phi_{0}(x) \mathcal{P}(q)(\omega, x) d \sigma d t \\
& =\int_{\omega^{\perp}} e^{-i x \cdot \xi} \phi_{0}(x) \mathcal{P}(q)(\omega, x) d \sigma .
\end{aligned}
$$

Then, by (3.30) and (3.18), we conclude that for any $\tau \geq \tau_{0}$ it holds that

$$
\left|\int_{\omega^{\perp}} e^{-i x \cdot \xi} \phi_{0}(x) \mathcal{P}(q)(\omega, x) d \sigma\right| \leq \tau^{3}\|\phi\|_{H^{2}}^{2}\left\|\Lambda_{A_{1}, q_{1}}-\Lambda_{A_{2}, q_{2}}\right\|+\frac{C}{\tau} \mathcal{N}_{\omega}(\phi)^{2}+C \tau\left\|A^{\prime}\right\|_{L^{\infty}} \mathcal{N}_{\omega}(\phi)^{2} .
$$

On the other hand, by (3.28), there is a constant $C>0$, which depends on $\|A\|_{W^{3, \infty}}$ such that

$$
\|\phi\|_{H^{2}\left(\mathbb{R}^{d}\right)} \leq C\langle\xi\rangle^{2}
$$

We conclude that by (3.28), there is a constant $C>0$ which depends also on $\|A\|_{W^{3, \infty}}$ such that for any $\xi \in \omega^{\perp}$ it holds that

$$
\mathcal{N}_{\omega}(\phi)^{2} \leq C\langle\xi\rangle^{4} .
$$

Thus, by (3.33)-(3.32), we conclude that for any $\tau \geq \tau_{0}$ and $\xi \in \omega^{\perp}$ it holds that

$$
\left|\int_{\omega^{\perp}} e^{-i x \cdot \xi} \mathcal{P}(q)(\omega, x) d \sigma\right| \leq C \tau^{3}\langle\xi\rangle^{4}\left\|\Lambda_{A_{1}, q_{1}}-\Lambda_{A_{2}, q_{2}}\right\|+\frac{C}{\tau}\langle\xi\rangle^{4}+C \tau\left\|A^{\prime}\right\|_{L^{\infty}}\langle\xi\rangle^{4}
$$

for some positive constant $C$ which depends on $\|A\|_{W^{3, \infty}}$. Consequently, by (3.34), (3.16), (3.10) and the result of Theorem 2, we see that for any $\tau \geq \tau_{0}, \mu \in(0,1)$ and $\xi \in \omega^{\perp}$ it holds that

$$
|\widehat{q}(\xi)| \leq C \tau^{2}\langle\xi\rangle^{4}\left\|\Lambda_{A_{1}, q_{1}}-\Lambda_{A_{2}, q_{2}}\right\|+\frac{C}{\tau}\langle\xi\rangle^{4}+C \tau\langle\xi\rangle^{4}\left\|\Lambda_{A_{1}, q_{1}}-\Lambda_{A_{2}, q_{2}}\right\|^{\mu} .
$$

changing $\omega \in \mathbb{S}^{d-1}$, then for any $\xi \in \mathbb{R}^{d}$, we get

$$
|\widehat{q}(\xi)| \leq C \tau^{3}\langle\xi\rangle^{4}\left\|\Lambda_{A_{1}, q_{1}}-\Lambda_{A_{2}, q_{2}}\right\|+\frac{C}{\tau}\langle\xi\rangle^{4}+C \tau\langle\xi\rangle^{4}\left\|\Lambda_{A_{1}, q_{1}}-\Lambda_{A_{2}, q_{2}}\right\|^{\mu} .
$$

This completes the proof of Lemma 3.5.

We will now complete the proof of Theorem 2. Using (3.27) we get

$$
\begin{aligned}
\|q\|_{H^{-1}\left(\mathbb{R}^{d}\right)}^{2} & =\int_{|\xi| \leq R}|\widehat{q}(\xi)|^{2}\langle\xi\rangle^{-2} d \xi+\int_{|\xi|>R}|\widehat{q}(\xi)|^{2}\langle\xi\rangle^{-2} d \xi \\
& \leq C\left(R^{d}\|\widehat{q}\|_{L^{\infty}(B(0, R))}^{2}+\frac{1}{R^{2}}\|q\|_{L^{2}(\Omega)}^{2}\right) \\
& \leq C\left(\tau^{6} R^{d+8}\left\|\Lambda_{A_{1}, q_{1}}-\Lambda_{A_{2}, q_{2}}\right\|^{2}+\tau^{2} R^{d+8}\left\|\Lambda_{A_{1}, q_{1}}-\Lambda_{A_{2}, q_{2}}\right\|^{\mu}+\frac{R^{d+8}}{\tau^{2}}+\frac{1}{R^{2}}\right) .
\end{aligned}
$$


Choosing

$$
\tau^{2}=R^{d+10}
$$

we obtain

$$
\|q\|_{H^{-1}\left(\mathbb{R}^{d}\right)} \leq C\left(R^{k}\left\|\Lambda_{A_{1}, q_{1}}-\Lambda_{A_{2}, q_{2}}\right\|+R^{k}\left\|\Lambda_{A_{1}, q_{1}}-\Lambda_{A_{2}, q_{2}}\right\|^{\mu}+\frac{1}{R}\right)
$$

for some positive constant $k$. The argument above are valid if $\tau \geq \tau_{0}$. By (3.37) we need to take $R$ sufficiently large. So there exists a $\gamma>0$ such that if $\left\|\Lambda_{A_{1}, q_{1}}-\Lambda_{A_{2}, q_{2}}\right\|<\gamma$ and $R=\left\|\Lambda_{A_{1}, q_{1}}-\Lambda_{A_{2}, q_{2}}\right\|^{-\frac{\mu}{k+1}}$ we have $\tau \geq \tau_{0}$ and by (3.38) we obtain

$$
\|q\|_{H^{-1}\left(\mathbb{R}^{d}\right)} \leq C\left\|\Lambda_{A_{1}, q_{1}}-\Lambda_{A_{2}, q_{2}}\right\|^{\mu^{\prime}}
$$

where $\mu^{\prime}=\mu /(k+1)$. Now if $\left\|\Lambda_{A_{1}, q_{1}}-\Lambda_{A_{2}, q_{2}}\right\| \geq \gamma$. Then we have

$$
\|q\|_{H^{-1}\left(\mathbb{R}^{d}\right)} \leq \frac{2 M}{\gamma^{1 /(k+1)}} \gamma^{1 /(k+1)} \leq \frac{2 M}{\gamma^{\mu}}\left\|\Lambda_{A_{1}, q_{1}}-\Lambda_{A_{2}, q_{2}}\right\|^{\mu} .
$$

This completes the proof of Theorem 2 .

\subsection{Proof of Theorem 3}

Using the expression of $f_{\tau}$ given by $e^{i \tau(x \cdot \omega+t)} \phi_{2}(x+t \cdot \omega) b_{2}(t, x)$ we have the following estimate

$$
\left\|\partial_{t}^{(k)} f_{\tau}\right\|_{L^{2}\left((0, T), H^{3 / 2}(\Gamma)\right)} \leq C\left(\tau^{k}\|\phi\|_{H^{2}\left(\mathbb{R}^{d}\right)}+\sum_{j=1}^{k} \tau^{k-j}\left\|(\omega \cdot \nabla)^{j} \phi\right\|_{H^{2}\left(\mathbb{R}^{d}\right)}\right),
$$

for any $k \in\{1, \ldots, 2 d+4\}$.

From (3.14),we have

$$
\begin{aligned}
\left|\int_{\Sigma}\left(\Lambda_{A_{1}, q_{1}}^{\sharp}-\Lambda_{A_{2}, q_{2}}^{\sharp}\right)\left(f_{\tau}\right)(t, x) \bar{g}_{\tau}(t, x) d \sigma_{x} d t\right| & \leq\left\|\left(\Lambda_{A_{1}, q_{1}}^{\sharp}-\Lambda_{A_{2}, q_{2}}^{\sharp}\right) f_{\tau}\right\|_{L^{2}(\Sigma)}\left\|g_{\tau}\right\|_{L^{2}(\Sigma)} \\
& \leq\left\|\Lambda_{A_{1}, q_{1}}^{\sharp}-\Lambda_{A_{2}, q_{2}}^{\sharp}\right\|_{s}\left\|f_{\tau}\right\|_{H^{2 d+4}\left(0, T, L^{2}(\Gamma)\right)}\left\|g_{\tau}\right\|_{L^{2}(\Sigma)} .
\end{aligned}
$$

Using the estimate (3.41), then we have

$$
\left\|f_{\tau}\right\|_{H^{2 d+4}\left(0, T ; H^{3 / 2}(\Gamma)\right)} \leq C\left(\tau^{2 d+4}\|\phi\|_{H^{2}\left(\mathbb{R}^{d}\right)}+\sum_{j=1}^{2 d+4} \tau^{(2 d+4)-j}\left\|(\omega \cdot \nabla)^{j} \phi\right\|_{H^{2}\left(\mathbb{R}^{d}\right)}\right) .
$$

On the other hand Using (3.28) we obtain

$$
\|\phi\|_{H^{2}\left(\mathbb{R}^{d}\right)} \leq\langle\xi\rangle^{2} \text { and }\left\|(\omega \cdot \nabla)^{j} \phi\right\|_{H^{2}\left(\mathbb{R}^{d}\right)} \leq\langle\xi\rangle^{2} \text { for } 1 \leq j \leq 2 d+4 .
$$

Then

$$
\left\|f_{\tau}\right\|_{H^{2 d+4}\left(0, T ; H^{3 / 2}(\Gamma)\right)} \leq C \tau^{2 d+4}\langle\xi\rangle^{2} .
$$

Finally, combining the precedent result, we can show the following estimate

$$
\left|\int_{\Sigma}\left(\Lambda_{A_{1}, q_{1}}^{\sharp}-\Lambda_{A_{2}, q_{2}}^{\sharp}\right)\left(f_{\tau}\right)(t, x) \bar{g}_{\tau}(t, x) d \sigma_{x} d t\right| \leq C \tau^{2 d+5}\langle\xi\rangle^{3} .
$$

The rest of the proof is similar to that of Theorem 2 and the proof given in article [3]. 


\section{A Appendix}

\section{A.1 Construction of solution with spectral data}

We are interested to study the form of the solution for the wave equation.

Lemma A.1 Let $\Omega \subset \mathbb{R}^{n}$ be an open bounded set, $I=(0, T)$ an interval include in $\mathbb{R}$. Let $F \in$ $C^{0}\left((0, T), L^{2}(\Omega)\right)$. There exists a unique solution $u \in C^{0}\left((0, T), H_{0}^{1}(\Omega)\right) \cap C^{0}\left((0, T), L^{2}(\Omega)\right)$ such that,

$$
\begin{cases}\partial_{t}^{2} u-\Delta_{A} u+q u=F(t, x) & \text { in } \quad(0, T) \times \Omega, \\ u(0, \cdot)=\partial_{t} u(0, \cdot)=0 & \text { in } \Omega, \\ u(t, x)=0 & \text { on } \Sigma\end{cases}
$$

This solution is given by

$$
u(t, x)=\sum_{k=1}^{\infty} u_{k}(t) \varphi_{k}(x)
$$

where

$$
u_{k}(t)=\int_{\Omega} \int_{0}^{t} F(\tau, y) s_{k}(t-\tau) \varphi_{k}(y) d \tau d y
$$

and

$$
s_{k}(t)=\left\{\begin{array}{cl}
\frac{\sin \sqrt{\lambda_{k}} t}{\sqrt{\lambda_{k}}}, & \text { if } \lambda_{k}>0, \\
t, & \text { if } \lambda_{k}=0, \\
\frac{\sin \sqrt{\lambda_{k}} t}{\sqrt{\lambda_{k}}}, & \text { if } \lambda_{k}<0 .
\end{array}\right.
$$

Proof . The Fourier expansion of the wave $u(t, x)$ is given by the formula

$$
u(t, x)=\sum_{k=1}^{\infty} u_{k}(t) \varphi_{k}(x),
$$

where $u_{k}$ is the Fourier coefficients. We want to determinate the expression of these coefficients, For that, we have

$$
\begin{aligned}
\frac{d^{2}}{d t^{2}} u_{k}(t) & =\int_{\Omega} \frac{d^{2}}{d t^{2}} u(t, x) \cdot \bar{\varphi}_{k}(x) d x \\
& =\int_{\Omega}\left(\Delta_{A}-q\right) u(t, x) \bar{\varphi}_{k}(x) d x+\int_{\Omega} F(t, x) \bar{\varphi}_{k}(x) d x \\
& =-\lambda_{k} \int_{\Omega} u(t, x) \bar{\varphi}_{k}(x) d x+\int_{\Omega} F(t, x) \bar{\varphi}_{k}(x) d x \\
& =-\lambda_{k} u_{k}(t)+\int_{\Omega} F(t, x) \bar{\varphi}_{k}(x) d x
\end{aligned}
$$

This equation is an ordinary differential equation for $u_{k}(t)$

$$
\frac{d^{2}}{d t^{2}} u_{k}(t)+\lambda_{k} u_{k}(t)=\int_{\Omega} F(t, x) \bar{\varphi}_{k}(x) d x .
$$


By using the standard resolution of the differential equation, we have

$$
\begin{aligned}
u_{k}(t, x) & =\int_{0}^{t} \frac{1}{\sqrt{\lambda_{k}}}\left[\cos \sqrt{\lambda_{k}} \tau \cdot \sin \sqrt{\lambda_{k}} t-\sin \sqrt{\lambda_{k}} \tau \cdot \cos \sqrt{\lambda_{k}} t\right]\left(\int_{\Omega} F(\tau, x) \bar{\varphi}_{k}(x) d x\right) d \tau \\
& =\int_{0}^{t} \frac{\sin \sqrt{\lambda_{k}}(t-\tau)}{\sqrt{\lambda_{k}}}\left(\int_{\Omega} F(\tau, x) \bar{\varphi}_{k}(x) d x\right) d \tau .
\end{aligned}
$$

This completes the proof of the Lemma.

Remark 3 In the general case,

if $u$ solving

$$
\begin{cases}-\Delta_{A} u+q u-\lambda u=0 & \text { in } \quad \Omega, \\ u(x)=f & \text { on } \quad \Gamma\end{cases}
$$

then $u$ is given by $u(x)=\sum_{k \geq 1} \frac{\varphi_{k}(x)}{\lambda_{k}-\lambda} \int_{\Gamma} \overline{\partial_{\nu_{y}} \varphi_{k}}(y) f(y) d S_{y}$.

If $v$ solving

$$
\begin{cases}-\Delta_{A} v+q v-\lambda v=F & \text { in } \quad \Omega, \\ v(x)=0 & \text { on } \quad \Gamma\end{cases}
$$

then $v$ is given by $v(x)=\sum_{k \geq 1} \frac{\varphi_{k}(x)}{\lambda_{k}-\lambda} \int_{\Omega} \overline{\varphi_{k}}(y) F(y) d y$.

\section{A.2 Estimates for $\Pi_{A, q}$ and its relation with $\Lambda_{A, q}^{\sharp}$}

Lemma A.2 Let $0<s<\frac{1}{2}$. We suppose that $j$ is fixed. Then, we have

$$
\left\|\left(\frac{d}{d \lambda}\right)^{j}\left(\Pi_{A_{1}, q_{1}}(\lambda)-\Pi_{A_{2}, q_{2}}(\lambda)\right)\right\|_{\frac{3}{2}, s} \leq C|\lambda|^{-j+\frac{1}{4}(1+2 s)} .
$$

To prove this Lemma, we will use the elliptic result given by

Lemma A.3 Let $v$ and $w$ are the solution of

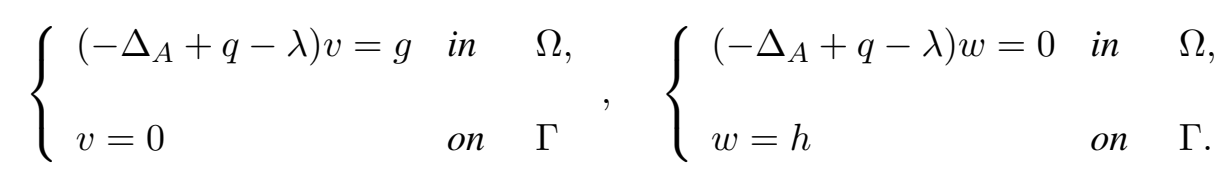

We have the following estimates,

$$
\begin{aligned}
& \|v\|_{H^{s}(\Omega)} \leq C|\lambda|^{s / 2-1}\|g\|_{L^{2}(\Omega)}, \quad 0 \leq s \leq 2 \\
& \|w\|_{L^{2}(\Omega)} \leq C\|h\|_{H^{1 / 2}(\Gamma)} \quad \text { and } \quad\|w\|_{H^{1}(\Omega)} \leq C\|h\|_{H^{1 / 2}(\Gamma)} .
\end{aligned}
$$


Proof of Lemma A.2. Let $u_{l}$ the solution of the system (2.1) and $G_{l}(\lambda, x, y)$ the Green function satisfy, for $l=1,2$

$$
\begin{cases}\left(-\Delta_{A_{l}}+q_{l}-\lambda\right) G(\lambda, ., y)=\delta(x-y) & (x, y) \in \Omega \times \Omega \\ G(\lambda, x, y)=0 & (x, y) \in \Gamma \times \Omega .\end{cases}
$$

We note by $\left\{\varphi_{k}^{l}, k \geq 1\right\}$, the eigenfunctions which satisfies

$$
\begin{cases}\left(-\Delta_{A_{l}}+q_{l}\right) \varphi_{k}^{l}=\lambda_{k}^{l} \varphi_{k}^{l} & \text { in } \Omega, \\ \varphi_{k}^{l}=0 & \text { on } \Gamma .\end{cases}
$$

Let $\left\{\varphi_{k}^{l}, k \geq 1\right\}$ be a corresponding complete set of orthonormal eigenfunctions in $L^{2}(\Omega)$ and the orthogonal in $H^{1}(\Omega)$. So we can show that

$$
G_{l}(\lambda, x, y)=\sum_{k=1}^{\infty} \frac{\varphi_{k}^{l}(x) \overline{\varphi_{k}^{l}}(y)}{\left(\lambda_{k}^{l}-\lambda\right)} .
$$

We note that $u_{l}^{0}$ is the solution of

$$
\begin{cases}\left(-\Delta_{A_{l}}+q_{l}-\lambda\right) u_{l}^{0}=0 & \text { in } \Omega, \\ u_{l}^{0}=f & \text { on } \Gamma .\end{cases}
$$

According to what is given above, we know that the solution $u_{l}^{0}$ is given by

$$
\begin{aligned}
u_{l}^{0}(x) & =\int_{\Gamma} \frac{\partial G_{l}}{\partial \nu_{y}} f(y) d S_{y} \\
& =\sum_{k=1}^{\infty} \frac{\varphi_{k}^{l}(x)}{\left(\lambda_{k}^{l}-\lambda\right)} \int_{\Gamma} \overline{\partial_{\nu_{y}} \varphi_{k}^{l}}(y) f(y) d S_{y}
\end{aligned}
$$

and that the D-to-N map $\Pi_{A_{l}, q_{l}}(f)=\frac{\partial u_{l}^{0}}{\partial \nu}+i A_{l} \nu \cdot u_{l}^{0}$. We consider $u_{l}^{1}$ the solution of the following boundary value problem

$$
\begin{cases}\left(-\Delta_{A_{l}}+q_{l}-\lambda\right) u_{l}^{1}=u_{l}^{0} & \text { in } \Omega, \\ u_{l}^{1}=0 & \text { on } \Gamma .\end{cases}
$$

First we will prove that $u_{l}^{1}=\frac{d}{d \lambda}\left(u_{l}^{0}\right)$. According to what is given above, we know that the solution $u_{l}^{1}$ is given by the following expression

$$
\begin{aligned}
u_{l}^{1}(x) & =\int_{\Omega} G(\lambda, x, y) u_{l}^{0}(y) d y \\
& =\sum_{k=1}^{\infty} \frac{\varphi_{k}^{l}(x)}{\left(\lambda_{k}^{l}-\lambda\right)} \int_{\Omega} \overline{\varphi_{k}^{l}}(y) u_{l}^{0}(y) d y .
\end{aligned}
$$


To obtain the desired result, it is necessary to find a relation between the two expressions: $\int_{\Omega} \overline{\varphi_{k}^{l}}(y) u_{l}^{0}(y) d y$ and $\int_{\Gamma} \overline{\partial_{\nu_{y}} \varphi_{k}^{l}}(y) f(y) d S_{y}$. For that, we notice that $\mathscr{H}_{A_{l}, q_{l}}\left(\varphi_{k}^{l}\right)=\lambda_{k}^{l} \varphi_{k}^{l}$, so

$$
\mathscr{H}_{A_{l},\left(q_{l}-\lambda\right)} \varphi_{k}^{l}=\left(\lambda_{k}^{l}-\lambda\right) \varphi_{k}^{l},
$$

as a consequence $\varphi_{k}^{l}=\frac{1}{\left(\lambda_{k}^{l}-\lambda\right)} \mathscr{H}_{A_{l},\left(q_{l}-\lambda\right)} \varphi_{k}^{l}$. By replacing the expression of $\varphi_{k}^{l}$ in $\int_{\Omega} \overline{\varphi_{k}^{l}}(y) u_{l}^{0}(y) d y$ and using the integration by part, we obtain

$$
\begin{aligned}
\int_{\Omega} \overline{\varphi_{k}^{l}}(y) u_{l}^{0}(y) d y & =\int_{\Omega} \frac{1}{\left(\lambda_{k}^{l}-\lambda\right)} \overline{\mathscr{H}_{A_{l},\left(q_{l}-\lambda\right)} \varphi_{k}^{l}}(y) u_{l}^{0}(y) d y \\
& =\frac{1}{\left(\lambda_{k}^{l}-\lambda\right)}\left(\int_{\Omega} \overline{\varphi_{k}^{l}}(y) \mathscr{H}_{A_{l},\left(q_{l}-\lambda\right)} u_{l}^{0}(y) d y-\int_{\Gamma} \overline{\partial_{\nu_{y}} \varphi_{k}^{l}}(y) f(y) d S_{y}\right) .
\end{aligned}
$$

Finally, we have

$$
\int_{\Omega} \overline{\varphi_{k}^{l}}(y) u_{l}^{0}(y) d y=-\frac{1}{\left(\lambda_{k}^{l}-\lambda\right)} \int_{\Gamma} \overline{\partial_{\nu_{y}} \varphi_{k}^{l}}(y) f(y) d S_{y}
$$

So

$$
\begin{aligned}
u_{l}^{1}(x) & =\sum_{k=1}^{\infty} \frac{\varphi_{k}^{l}(x)}{\left(\lambda_{k}^{l}-\lambda\right)} \int_{\Omega} \overline{\varphi_{k}^{l}}(y) u_{l}^{0}(y) d y \\
& =-\sum_{k=1}^{\infty} \frac{\varphi_{k}^{l}(x)}{\left(\lambda_{k}^{l}-\lambda\right)^{2}} \int_{\Gamma} \overline{\partial_{\nu_{y}} \varphi_{k}^{l}}(y) f(y) d S_{y} \\
& =\frac{d}{d \lambda}\left(u_{l}^{0}\right) .
\end{aligned}
$$

We have

$$
\left(\frac{d}{d \lambda}\right)^{j} \Pi_{A_{l}, q_{l}}(\lambda)(f)=\left.\left(\frac{\partial}{\partial \nu}+i A_{l} . \nu\right) u_{l}^{j}\right|_{\Gamma}
$$

where $u_{l}^{j}$ is the solution of

$$
\begin{cases}\left(-\Delta_{A_{l}}+q_{l}-\lambda\right) u_{l}^{j}=u_{l}^{j-1} & \text { in } \quad \Omega, \\ u_{l}^{j}=0 & \text { on } \quad \Gamma, \text { for } j \geq 1 .\end{cases}
$$

We remark that, for $j \geq 1$, we have $\left(\frac{d}{d \lambda}\right)^{j} \Pi_{A_{l}, q_{l}}(\lambda)(f)=\left.\left(\frac{\partial}{\partial \nu}\right) u_{l}^{j}\right|_{\Gamma}$.

Let $v^{j}=u_{1}^{j}-u_{2}^{j}$, It is easy to show that

$$
\left(\frac{d}{d \lambda}\right)^{j}\left(\Pi_{A_{1}, q_{1}}(\lambda)-\Pi_{A_{2}, q_{2}}(\lambda)\right)(f)=\left.\left(\frac{\partial}{\partial \nu}\right) v^{j}\right|_{\Gamma}, \quad \text { for } j \neq 0 .
$$

We note that $v^{0}$ is the solution of

$$
\begin{cases}\left(-\Delta_{A_{1}}+q_{1}-\lambda\right) v^{0}=2 i A(x) \cdot \nabla u_{2}^{0}+V(x) u_{2}^{0} & \text { in } \quad \Omega \\ v^{0}=0 & \text { on } \Gamma\end{cases}
$$


where $V(x)=V_{1}(x)-V_{2}(x), V_{l}=i \operatorname{div}\left(A_{l}\right)-A_{l} \cdot A_{l}-q_{l}$, for $l=1,2$ and $v^{j}$ satisfied, for $j \geq 1$, the following problem

$$
\begin{cases}\left(-\Delta_{A_{1}}+q_{1}-\lambda\right) v^{j}=2 i A(x) \cdot \nabla u_{2}^{j}+V(x) u_{2}^{j}+v^{j-1} & \text { in } \quad \Omega, \\ v^{j}=0 & \text { on } \quad \Gamma .\end{cases}
$$

Using lemma A.3, we have for $0 \leq s \leq 2$ and $j \geq 1$, the following estimates

$$
\left\|u_{l}^{j}\right\|_{H^{s}(\Omega)} \leq|\lambda|^{s / 2-1}\left\|u_{l}^{j-1}\right\|_{L^{2}(\Omega)} \text { and } \quad\left\|u_{l}^{j-1}\right\|_{L^{2}(\Omega)} \leq C|\lambda|^{-(j-1)}\|f\|_{H^{1 / 2}(\Gamma)} .
$$

So

$$
\left\|u_{l}^{j}\right\|_{H^{s}(\Omega)} \leq|\lambda|^{s / 2-j}\|f\|_{H^{1 / 2}(\Gamma)} .
$$

Using the result (A.4) to the solution $v^{j}$, we have

$$
\left\|v^{j}\right\|_{H^{s}(\Omega)} \leq|\lambda|^{s / 2-1}\left\|u_{2}^{j}\right\|_{H^{1}(\Omega)}+|\lambda|^{s / 2-1}\left\|v^{j-1}\right\|_{L^{2}(\Omega)} .
$$

Substituting (A.17) in (A.18), we obtain

$$
\left\|v^{j}\right\|_{H^{s}(\Omega)} \leq|\lambda|^{(s-1) / 2-j}\|f\|_{H^{1 / 2}(\Gamma)}+|\lambda|^{s / 2-1}\left\|v^{j-1}\right\|_{L^{2}(\Omega)} .
$$

According to (A.4), we have the following estimate

$$
\begin{aligned}
\left\|v^{j-1}\right\|_{L^{2}(\Omega)} & \leq|\lambda|^{-1}\left\|u_{2}^{j-1}\right\|_{H^{1}(\Omega)}+|\lambda|^{-1}\left\|v^{j-2}\right\|_{L^{2}(\Omega)} \\
& \leq|\lambda|^{(1 / 2-j)}\|f\|_{H^{1 / 2}(\Gamma)}+|\lambda|^{-1}\left\|v^{j-2}\right\|_{L^{2}(\Omega)} .
\end{aligned}
$$

We repeat the same work, we show that, for $j \geq 1$, we have

$$
\left\|v^{j}\right\|_{H^{s}(\Omega)} \leq C|\lambda|^{(s-1) / 2-j}\|f\|_{H^{1 / 2}(\Gamma)} \quad 0 \leq s \leq 2,
$$

where $C$ depend only on $\Omega$.

So, by theorem of traces, we have for $j \geq 1$

$$
\begin{aligned}
\left\|\frac{\partial}{\partial \nu} v^{j}\right\|_{H^{s}(\Gamma)} & \leq C\left\|v^{j}\right\|_{H^{s+3 / 2}(\Omega)} \\
& \leq|\lambda|^{(1+2 s) / 4-j}\|f\|_{H^{1 / 2}(\Gamma)}
\end{aligned}
$$

and

$$
\begin{aligned}
\left\|v^{0}\right\|_{H^{s}(\Gamma)} & \leq C\left\|v^{0}\right\|_{H^{1 / 2+s}(\Omega)} \\
& \leq|\lambda|^{-1 / 4+s / 2}\|f\|_{H^{1 / 2}(\Gamma)} \leq|\lambda|^{-(1-2 s) / 4}\|f\|_{H^{1 / 2}(\Gamma)} .
\end{aligned}
$$

This completes the proof of the Lemma. 
Lemma A.4 For all $f \in H^{2(d+2)}\left(0, T, H^{1 / 2}(\Gamma)\right)$ satisfies

$$
\left(\frac{\partial}{\partial t}\right)^{j} f(0, .)=0 \text { pour } j=0,1, \ldots, 2 d+3 .
$$

We have

$$
\Lambda_{A, q}^{\sharp}(f)=\sum_{j=0}^{d+1}\left[\left(\frac{d}{d \lambda}\right)^{j} \Pi_{A, q}(\lambda)\right]_{\lambda=0}\left(-\frac{\partial^{2}}{\partial t^{2}}\right)^{j} f+\mathscr{R}_{A, q}(f),
$$

where

$$
\mathscr{R}_{A, q}(f)=\sum_{k=1}^{\infty}\left(\lambda_{k}\right)^{-d-5 / 2} \frac{\partial \varphi_{k}}{\partial \nu} \int_{0}^{t} \sin \sqrt{\lambda_{k}}(t-s)\left(\int_{\Gamma}\left(-\frac{\partial^{2}}{\partial s^{2}}\right)^{d+2} f(s, y) \overline{\partial_{\nu_{y}} \varphi_{k}}(y) d S_{y}\right) d s+i A . \nu f .
$$

Proof. We consider solution $u$ of the system

$$
\begin{cases}\partial_{t}^{2} u(t, x)-\Delta_{A} u(t, x)+q(x) u(t, x)=0 & \text { in } Q=(0, T) \times \Omega \\ u(0, \cdot)=\partial_{t} u(0, \cdot)=0 & \text { in } \Omega \\ u(t, x)=f & \text { on } \Sigma\end{cases}
$$

Let $u$ the solution given by the following expression

$$
u(t, x)=\sum_{j=0}^{d+1} u^{j}(t, x)+h(t, x)
$$

where $u^{0}$ satisfy

$$
\begin{cases}\Delta_{A} u^{0}(t, x)+q(x) u^{0}(t, x)=0 & \text { in } \quad Q \\ u^{0}(t, x)=f & \text { on } \quad \Sigma .\end{cases}
$$

For all $1 \leq j \leq d+1$, we have $u^{j}$ verify

$$
\begin{cases}\Delta_{A} u^{j}(t, x)+q(x) u^{j}(t, x)=\left(-\frac{\partial^{2}}{\partial t^{2}}\right) u^{j-1}(t, x) & \text { in } \quad Q \\ u^{j}(t, x)=0 & \text { on } \Sigma .\end{cases}
$$

and $h$ the solution of

$$
\begin{cases}\partial_{t}^{2} h(t, x)-\Delta_{A} h(t, x)+q(x) h(t, x)=\left(-\frac{\partial^{2}}{\partial t^{2}}\right) u^{d+1}(t, x) & \text { in } \quad Q \\ h(0, x)=\partial_{t} h(0, x)=0 & \text { in } \Omega \\ h(t, x)=0 & \text { on } \Sigma .\end{cases}
$$

Using the remak 3 , we obtain the expression of $u^{0}$ and $u^{1}$ given by

$$
u^{0}(t, x)=\left.\sum_{k \geq 1} \frac{\varphi_{k}(x)}{\lambda_{k}-\lambda} \int_{\Gamma} \overline{\partial_{\nu_{y}} \varphi_{k}}(y) f(t, y) d S_{y}\right|_{\lambda=0} .
$$




$$
u^{1}(t, x)=\left.\sum_{k \geq 1} \frac{\varphi_{k}(x)}{\lambda_{k}-\lambda} \int_{\Omega} \overline{\varphi_{k}}(y)\left(-\frac{\partial^{2}}{\partial t^{2}}\right) u^{0}(t, y) d y\right|_{\lambda=0} .
$$

Furthermore, we know that $\varphi_{k}=\frac{1}{\lambda_{k}} \mathscr{H}_{A, q} \varphi_{k}$, so by integrating by part, we have

$$
\int_{\Omega} \overline{\varphi_{k}}(y)\left(-\frac{\partial^{2}}{\partial t^{2}}\right) u^{0}(t, y) d y=-\frac{1}{\lambda_{k}-\lambda} \int_{\Gamma} \overline{\varphi_{k}}(y)\left(-\frac{\partial^{2}}{\partial t^{2}}\right) f(t, y) d S_{y} .
$$

Thus

$$
u^{1}(t, x)=-\sum_{k \geq 1} \frac{\varphi_{k}(x)}{\left(\lambda_{k}-\lambda\right)^{2}} \int_{\Gamma} \overline{\varphi_{k}}(y)\left(-\frac{\partial^{2}}{\partial t^{2}}\right) f(t, y) d S_{y} .
$$

By applying the lemma A.1, where $F(t, x)=\left(-\frac{\partial^{2}}{\partial t^{2}}\right) u^{d+1}(t, x), h$ is given by

$$
h(t, x)=\sum_{k \geq 1} \varphi_{k}(x)\left[\int_{0}^{t} \frac{\sin \sqrt{\lambda_{k}}(t-s)}{\sqrt{\lambda_{k}}}\left(\int_{\Omega}\left(-\frac{\partial^{2}}{\partial s^{2}}\right) u^{d+1}(s, y) \overline{\varphi_{k}}(y) d y\right) d s\right] .
$$

Now we will show that

$$
\int_{\Omega}\left(-\frac{\partial^{2}}{\partial t^{2}}\right) u^{d+1}(t, y) \overline{\varphi_{k}}(y) d y=\left(\lambda_{k}\right)^{-(d+2)} \int_{\Gamma}\left(-\frac{\partial^{2}}{\partial t^{2}}\right)^{(d+2)} f \overline{\partial_{\nu_{y}} \varphi_{k}}(y) d S_{y} .
$$

Indeed, we know that $u^{d+1}$ solving the following equation

$$
\begin{cases}\Delta_{A} u^{d+1}+q u^{d+1}=\left(-\frac{\partial^{2}}{\partial t^{2}}\right) u^{d} & \text { in } \quad Q \\ u^{d+1}(t, x)=0 & \text { on } \quad \Sigma .\end{cases}
$$

Furthermore $\varphi_{k}=\lambda_{k}^{-(d+2)}\left(\mathscr{H}_{A, q}\right)^{(d+2)} \varphi_{k}$, so we can rewrite the expression $\int_{\Omega}\left(-\frac{\partial^{2}}{\partial t^{2}}\right) u^{d+1} \overline{\varphi_{k}} d x$ as follows

$$
\begin{aligned}
\int_{\Omega}\left(-\frac{\partial^{2}}{\partial t^{2}}\right) u^{d+1} \overline{\varphi_{k}} d x & =\lambda_{k}^{d+2} \int_{\Omega}\left(-\frac{\partial^{2}}{\partial t^{2}}\right) u^{d+1} \overline{\left(\mathscr{H}_{A, q}\right)^{(d+2)} \varphi_{k}} d x \\
& =\lambda_{k}^{d+2} \int_{\Omega}\left(-\frac{\partial^{2}}{\partial t^{2}}\right)\left(\left(-\frac{\partial^{2}}{\partial t^{2}}\right) u^{d}\right) \overline{\left(\mathscr{H}_{A, q}\right)^{(d+1)} \varphi_{k}} d x
\end{aligned}
$$

we repeat the same calculus, we find

$$
\lambda_{k}^{d+2} \int_{\Omega}\left(-\frac{\partial^{2}}{\partial t^{2}}\right)\left(\left(-\frac{\partial^{2}}{\partial t^{2}}\right) u^{d}\right) \overline{\left(\mathscr{H}_{A, q}\right)^{(d+1)} \varphi_{k}} d x=\lambda_{k}^{d+2} \int_{\Omega}\left(-\frac{\partial^{2}}{\partial t^{2}}\right)^{(3)} u^{d \overline{\left(\mathscr{H}_{A, q}\right)^{(d+1)} \varphi_{k}}} d x .
$$

Finally, we have

$$
\lambda_{k}^{d+2} \int_{\Omega}\left(-\frac{\partial^{2}}{\partial t^{2}}\right) u^{d+1} \overline{\left(\mathscr{H}_{A, q}\right)^{(d+2)} \varphi_{k}} d x=\int_{\Gamma}\left(-\frac{\partial^{2}}{\partial t^{2}}\right)^{(d+2)} f(t, y) \cdot \overline{\partial_{\nu_{y}} \varphi_{k}}(y) d S_{y} .
$$

Therefore

$$
h(t, x)=\int_{0}^{t} \int_{\Gamma} \frac{\partial}{\partial \nu_{y}} \Gamma(t-s, x, y)\left(-\frac{\partial^{2}}{\partial s^{2}}\right)^{(d+2)} f(s, y) d S_{y} d s
$$


where

$$
\Gamma(t, x, y)=\sum_{k=1}^{\infty}\left(\lambda_{k}\right)^{-d-5 / 2} \varphi_{k}(x) \cdot \overline{\varphi_{k}}(y) \sin \sqrt{\lambda_{k}} t
$$

\section{References}

[1] G.Alessandrini and J.Sylvester, Stabitity for multidimensional inverse spectral problem, Comm . Partial Differential Equations 15 (5) (1990), 711-736.

[2] M. Bellassoued, Uniqueness and stability in determining the speed of propagation of second-order hyperbolic equation with variable coefficients, Applicable Analysis 83 (2004), 983-1014.

[3] M. Bellassoued and H.Ben Joud, Stability estimate for an inverse problem for the wave equation in a magnetic field, Applicable Analysis 87 (3) (2008), 277-292.

[4] M. Bellassoued, M. Choulli and M. Yamamoto, Stability estimate for an inverse wave equation and a multidimensional Borg-Levinson Theorem, J. Diff. Equat. 247 (2) (2009), 465-494.

[5] M.Bellassoued, D.Jellali and M.Yamamoto, Lipschitz stability for a hyperbolic inverse problem by finite local boundary data, Applicable Analysis 85 (2006), 1219-1243.

[6] M.Bellassoued, D.Jellali and M.Yamamoto, Stability Estimate for the hyperbolic inverse boundary value problem by local Dirichlet-to-Neumann map, J.Math.Anal.Appl. 343 (2) (2008), 1036-1046.

[7] H.Ben Joud, A stability estimate for an inverse problem for the Schrödinger equation in a magnetic field from partial boundary measurements, Inverse Problems 25, Issue 4, (2009) doi: 10.1088/02665611/25/4/045012.

[8] Y.M. Berezanskii, On the unique determination of a Schrödinger operator via its spectral function, Dokl. Akad. Nauk SSSR (In Russian). 93 (4) (1953), 197-200.

[9] Y.M. Berezanskii, Inverse spectral problem for Schrödinger operator, Dokl. Akad. Nauk SSSR (In Russian). 105 (2) (1955), 591-594-200.

[10] G. Börg, Umkehrung der Strum-Liouvillischen Eigenwertaufgabe. Bestimmung der Differentalgleichug durch die Eigenwerte, Acta Math. 78 (1) (1946), 1-96.

[11] M.Choulli Une introduction aux Problèmes Inverses Elliptiques et Paraboliques, Mathmatiques et Applications, Vol. 65, Springer-Verlag, Berlin.

[12] G.Eskin, A new approach to hyperbolic inverse problems, arXiv:math/0505452v3 [math.AP], 2006.

[13] G.Eskin, Global uniqueness in the inverse scattering problem for the Schrödinger operator with external Yang-Mills potentials, Comm. Math. Phys. 222, (3), (2001), 503531.

[14] I.M. Gelfand and B.M. Levitan,On identification of the differential expression via its spectral function, Izv.Akad.Nauk SSSR(in Russian), Sek.Matem. 15, (4) (1951). 
[15] G.M. Henkin and R.G. Novikov, $A \bar{\partial}-$ equation in the multidimensional inverse scattering problem , Uspekh.Matem.Nauk SSSR(in Russian). 42 (1) (1987), 93-152.

[16] O.Yu.Imanuvilov and M.Yamamoto, Global uniqueness and stability in determining coefficients of wave equations, Comm. Partial Diff. Equations 26 (2001), 1409-1425.

[17] V.Isakov, An inverse hyperbolic problem with many boundary measurements, Comm. Part. Dif. Equations 16 (1991), 1183-1195.

[18] V.Isakov, Inverse Problems for Partial Differential Equations, Springer-Verlag, Berlin, (1998).

[19] V. Isakov and Z. Sun, Stability estimates for hyperbolic inverse problems with local boundary data, Inverse Problems 8 (1992), 193-206.

[20] I.S. Kac and M.G. Krein, Spectral function of a string, In F. Atkinson; Discrete and Continous Boundary Value Problems (In Russian). Mir, Moskow (1968), 749 pp.

[21] M.G. Krein, The transmission function of the second-order one dimensional boundary value problem, Dokl. Akad. Nauk SSSR (In Russian). 88 (3) (1953), 405-408.

[22] A. Katchalov, Y. Kurylev and M. Lassas, Inverse Boundary Spectral Problems, Chapman \& Hall/CRC, Boca Raton, (2001).

[23] N.Levinson, The inverse Strum-Liouville problem, Mat.Tidsskr.B, (1949),25-30.

[24] J-L. Lions and E. Magenes, Non-homogenous Boundary Value Problems and Applications, Volumes I and II, Springer-Verlag, Berlin, (1972).

[25] A.Nachman, J.Sylvester and G.Uhlmann, An n-Dimensional Borg-Levinson Theorem, Comm. Math. Phys, 115 (1988), 595-605.

[26] F. Natterer, The Mathematics of Computarized Tomography, John Wiley \& Sons, Chichester, (1986).

[27] Rakesh, Reconstruction for an inverse problem for the wave equation with constant velocity, Inverse Problems 6 (1990), 91-98.

[28] Rakesh and W. Symes, Uniqueness for an inverse problems for the wave equation, Comm. Partial Diff. Equations 13 (1988), 87-96.

[29] A. Ramm and J. Sjöstrand, An inverse problem of the wave equation, Math. Z. 206 (1991), 119-130.

[30] A.Reiko and M.Sigeru: Propriétés asymptotiques des valeurs propres des opérateurs elliptiques auto-adjoints, J.Math.Kyoto Univ 4-1, (1964), 245-254.

[31] P. Stefanov and G. Uhlmann, Stability estimates for the hyperbolic Dirichlet to Neumann map in anisotropic media, J. Functional Anal. 154 (1998), 330-358.

[32] Z.Sun, On continous dependence for an inverse initial boundary value problem for the wave equation, J. Math. Anal. App. 150 (1990), 188-204.

[33] J.Sylvester and G.Uhlmann, A global uniqueness theorem for inveres boundary value problem, Ann.of Math.125, (2), (1987), 153-169. 
[34] L. Tzou, Stability estimates for coefficients of magnetic Schrodinger equation from full and partial boundary measurements, arXiv:math.AP/0602147. 